\title{
BALANCING DOMAIN DECOMPOSITION FOR NONCONFORMING PLATE ELEMENTS
}

\author{
Susanne C. Brenner* and Li-yeng Sung
}

Summary In this paper the balancing domain decomposition method is extended to nonconforming plate elements. The condition number of the preconditioned system is shown to be bounded by $C[1+\ln (H / h)]^{2}$, where $H$ measures the diameters of the subdomains, $h$ is the mesh size of the triangulation, and the constant $C$ is independent of $H, h$ and the number of subdomains.

Mathematics Subject Classification (1991): 65N55, 65N30

\section{Introduction}

Let $\Omega$ be a polygonal domain in $\mathbb{R}^{2}$. The variational form for the plate bending problem is given by (cf. [14])

$$
a(v, w)=\int_{\Omega}\left[\Delta v \Delta w+(1-\nu)\left(2 v_{x_{1} x_{2}} w_{x_{1} x_{2}}-v_{x_{1} x_{1}} w_{x_{2} x_{2}}-v_{x_{2} x_{2}} w_{x_{1} x_{1}}\right)\right] d x
$$

where the Poisson ratio $\nu$ satisfies $0<\nu<1 / 2$. The theory developed in this paper can be applied to the plate bending problem with general boundary conditions. However, for simplicity we only state the results for the clamped plate. The variational problem for the clamped plate is:

Find $u \in H_{0}^{2}(\Omega)$ such that

$$
a(u, v)=\int_{\Omega} f v d x \quad \forall v \in H_{0}^{2}(\Omega)
$$

where $u$ is the displacement and $f \in L^{2}(\Omega)$ is the body force.

Some of the simplest plate elements such as the Morley element (cf. [29]), the Zienkiewicz element (cf. [3]), the Fraeijs de Veubeke element (cf. [22]), the incomplete biquadratic element (cf. [34]) and the Adini element (cf. [1]) are nonconforming. Overlapping domain decomposition methods for nonconforming plate elements were studied in [9],

* The work of this author was supported in part by the National Science Foundation under Grant No. DMS-96-00133. 
[10], [11] and [12]. A nonoverlapping BPS-type algorithm for nonconforming plate elements was developed in [35]. In this paper we will extend Mandel's balancing domain decomposition (BDD) method (cf. [27], [28], [25], [26], [17], and for related algorithms, [20]), which is nonoverlapping, to nonconforming plate elements. The results will be presented in terms of the Morley element, but of course similar results hold for other nonconforming plate elements. Note that the BDD method for nonconforming finite elements of Lagrange type was studied in [16] for second order problems.

Let $\mathcal{T}_{h}$ be a quasi-uniform triangulation of $\Omega$ (cf. [13]) and $h=\max _{T \in \mathcal{T}_{h}}$ diamT. The Morley finite element space is $\mathcal{M}_{h}(\Omega)=\left\{v \in L^{2}(\Omega):\left.v\right|_{T} \in \mathcal{P}_{2}(T) \quad \forall T \in \mathcal{T}_{h}, v\right.$ is continuous at the vertices and vanishes at the vertices along $\partial \Omega$, the normal derivative of $v$ is continuous at the midpoints of interelement boundaries and vanishes at the midpoints along $\partial \Omega\}$. The discretization of $(1.2)$ using $\mathcal{M}_{h}(\Omega)$ is:

Find $u_{h} \in \mathcal{M}_{h}(\Omega)$ such that

$$
a_{h}\left(u_{h}, v\right)=\int_{\Omega} f v d x \quad \forall v \in \mathcal{M}_{h}(\Omega)
$$

where

$$
a_{h}(v, w)=\sum_{T \in \mathcal{T}_{h}} \int_{T}\left[\Delta v \Delta w+(1-\nu)\left(2 v_{x_{1} x_{2}} w_{x_{1} x_{2}}-v_{x_{1} x_{1}} w_{x_{2} x_{2}}-v_{x_{2} x_{2}} w_{x_{1} x_{1}}\right)\right] d x
$$

for all $v, w \in \mathcal{M}_{h}(\Omega)$. It follows from a simple calculation that

$$
\begin{aligned}
\left|a_{h}(v, w)\right| \leq(1+\nu)\left(\sum_{T \in \mathcal{T}_{h}}|v|_{H^{2}(T)}^{2}\right)^{1 / 2}\left(\sum_{T \in \mathcal{T}_{h}}|w|_{H^{2}(T)}^{2}\right)^{1 / 2} & \forall v, w \in \mathcal{M}_{h}(\Omega), \\
a_{h}(v, v) \geq(1-\nu) \sum_{T \in \mathcal{T}_{h}}|v|_{H^{2}(T)}^{2} & \forall v \in \mathcal{M}_{h}(\Omega),
\end{aligned}
$$

where $|v|_{H^{2}(T)}^{2}=\int_{T}\left[\left(v_{x_{1} x_{1}}\right)^{2}+2\left(v_{x_{1} x_{2}}\right)^{2}+\left(v_{x_{2} x_{2}}\right)^{2}\right] d x$.

Let $\Omega$ be partitioned into nonoverlapping polygonal subdomains $\Omega_{1}, \ldots, \Omega_{J}$, which are aligned with the triangulation $\mathcal{T}_{h}$ (cf. Figure 1 ). The subdomains $\Omega_{j}$ are assumed to be shape regular (the precise definition is stated in Section 4 ), and $H=\max _{1 \leq j \leq J} \operatorname{diam} \Omega_{\mathrm{j}}$. On the other hand, the $\Omega_{j}$ 's do not necessarily form a triangulation of $\Omega$. In other words, some of the cross points (i.e., points in $\Omega$ that belong to the boundary of at least three subdomains) may not be vertices of the subdomains (cf. Figure 1).

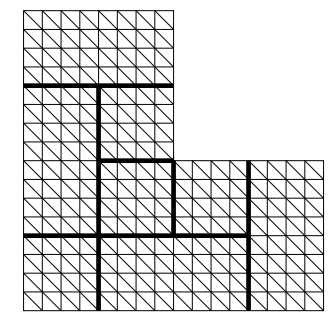

Figure 1 
Let $\Gamma_{j}=\partial \Omega_{j} \backslash \partial \Omega, \Gamma=\cup_{j=1}^{J} \Gamma_{j}, \mathcal{M}_{h}(\Omega \backslash \Gamma)$ be the subspace of members of $\mathcal{M}_{h}(\Omega)$ that vanish at all of the nodes on $\Gamma$, and $\mathcal{M}_{h}(\Gamma) \subseteq \mathcal{M}_{h}(\Omega)$ be the $a_{h}(\cdot, \cdot)$-orthogonal complement of $\mathcal{M}_{h}(\Omega \backslash \Gamma)$. We can write $u_{h}=\dot{u}_{h}+\bar{u}_{h}$, where $\dot{u}_{h} \in \mathcal{M}_{h}(\Omega \backslash \Gamma)$ and $\bar{u}_{h} \in \mathcal{M}_{h}(\Gamma)$. Problem (1.3) is equivalent to:

Find $\dot{u}_{h} \in \mathcal{M}_{h}(\Omega \backslash \Gamma)$ and $\bar{u}_{h} \in \mathcal{M}_{h}(\Gamma)$ such that

$$
\begin{array}{ll}
a_{h}\left(\dot{u}_{h}, v\right)=\int_{\Omega} f v d x & \forall v \in \mathcal{M}_{h}(\Omega \backslash \Gamma), \\
a_{h}\left(\bar{u}_{h}, v\right)=\int_{\Omega} f v d x & \forall v \in \mathcal{M}_{h}(\Gamma) .
\end{array}
$$

Let $\mathcal{M}_{h}\left(\Omega_{j} \cup \Gamma_{j}\right)$ be the restriction of $\mathcal{M}_{h}(\Omega)$ to $\Omega_{j}, \mathcal{M}_{h}\left(\Omega_{j}\right)$ be the subspace of $\mathcal{M}_{h}\left(\Omega_{j} \cup \Gamma_{j}\right)$ whose members vanish at all of the nodes on $\partial \Omega_{j}$, and $\mathcal{M}_{h}\left(\Gamma_{j}\right) \subseteq \mathcal{M}_{h}\left(\Omega_{j} \cup \Gamma_{j}\right)$ be the $a_{h, j}(\cdot, \cdot)$-orthogonal complement of $\mathcal{M}_{h}\left(\Omega_{j}\right)$, where $a_{h, j}(\cdot, \cdot)$ is the restriction of $a_{h}(\cdot, \cdot)$ to $\mathcal{M}_{h}\left(\Omega_{j} \cup \Gamma_{j}\right) \times \mathcal{M}_{h}\left(\Omega_{j} \cup \Gamma_{j}\right)$. A function $v \in \mathcal{M}_{h}\left(\Gamma_{j}\right)$ is called a discrete biharmonic Morley function and has the following minimum energy property:

$$
a_{h, j}(v, v) \leq a_{h, j}(w, w)
$$

for all $w \in \mathcal{M}_{h}\left(\Omega_{j} \cup \Gamma_{j}\right)$ that share the same nodal values as $v$ on $\Gamma_{j}$.

Since $\dot{u}_{h, j}=\left.\dot{u}_{h}\right|_{\Omega_{j}} \in \mathcal{M}_{h}\left(\Omega_{j}\right)$, we can find $\dot{u}_{h}$ by solving the following problems: Find $\dot{u}_{h, j} \in \mathcal{M}_{h}\left(\Omega_{j}\right)$ such that

$$
a_{h, j}\left(\dot{u}_{h, j}, v\right)=\int_{\Omega_{j}} f v d x \quad \forall v \in \mathcal{M}_{h}\left(\Omega_{j}\right) .
$$

Note that (1.6) applied to $\Omega_{j}$ implies that $a_{h, j}(\cdot, \cdot)$ is symmetric positive-definite on $\mathcal{M}_{h}\left(\Omega_{j}\right)$.

Since the subdomain problems (1.10) can be solved in parallel, the purpose of a nonoverlapping domain decomposition method is to provide an efficient solver for (1.8), which is the Schur complement of (1.3). Note that the Schur complement is very illconditioned: its condition number grows at a rate of $h^{-3}$ (cf. Section 7). The BDD method will produce a preconditioner for (1.8) so that the condition number of the preconditioned system is bounded by $C[1+\ln (H / h)]^{2}$.

The rest of this paper is organized as follows. The BDD algorithm is described in Section 2. The estimate for the condition number is based on the relation between the Morley element and the Hsieh-Clough-Tocher element, the equivalence of the nonconforming energy norm and a trace norm on the space $\mathcal{M}_{h}\left(\Gamma_{j}\right)$, and an extension estimate for quadratic functions in one variable involving a fractional order Sobolev norm. These are covered in Sections 3, 4 and 5 respectively. The theorem on the condition number estimate is given in Section 6, and some numerical results are reported in Section 7. 


\section{The BDD Method}

By the definitions of $\mathcal{M}_{h}(\Gamma)$ and $\mathcal{M}_{h}\left(\Gamma_{j}\right)$, the restriction operator $\mathbf{R}_{j}: \mathcal{M}_{h}(\Omega) \longrightarrow$ $\mathcal{M}_{h}\left(\Omega_{j} \cup \Gamma_{j}\right)$ defined by

$$
\mathbf{R}_{j} v=\left.v\right|_{\Omega_{j}}
$$

maps $\mathcal{M}_{h}(\Gamma)$ into $\mathcal{M}_{h}\left(\Gamma_{j}\right)$.

Note that the functions in $\mathcal{M}_{h}\left(\Gamma_{j}\right)$ are uniquely determined by their nodal values on $\Gamma_{j}$. In fact, for a given $v \in \mathcal{M}_{h}\left(\Gamma_{j}\right)$, if $w$ is any function in $\mathcal{M}_{h}\left(\Omega_{j} \cup \Gamma_{j}\right)$ that shares the same nodal values as $v$ on $\Gamma_{j}$, then $v-w \in \mathcal{M}_{h}\left(\Omega_{j}\right)$ is determined by

$$
a_{h, j}(v-w, z)=-a_{h, j}(w, z) \quad \forall z \in \mathcal{M}_{h}\left(\Omega_{j}\right)
$$

Since $\mathbf{R}_{j}$ maps $\mathcal{M}_{h}(\Gamma)$ into $\mathcal{M}_{h}\left(\Gamma_{j}\right)$, functions in $\mathcal{M}_{h}(\Gamma)$ are also determined by their nodal values on $\Gamma$. We can therefore define an extension map $\mathbf{E}_{j}: \mathcal{M}_{h}\left(\Gamma_{j}\right) \longrightarrow \mathcal{M}_{h}(\Gamma)$ as follows. Given any $v \in \mathcal{M}_{h}\left(\Gamma_{j}\right), \mathbf{E}_{j} v \in \mathcal{M}_{h}(\Gamma)$ shares the same values as $v$ at the nodes on $\Gamma_{j}$ and vanishes at all of the nodes on $\Gamma \backslash \Gamma_{j}$.

The operators $\mathbf{R}_{j}$ and $\mathbf{E}_{j}$ are related by

$$
\mathbf{R}_{j} \mathbf{E}_{j} v=v \quad \forall v \in \mathcal{M}_{h}\left(\Gamma_{j}\right)
$$

Let $V$ be a finite dimensional vector space and $V^{\prime}$ its dual. We will denote by $\langle\cdot, \cdot\rangle$ the canonical bilinear form between $V^{\prime}$ and $V$, i.e., if $F \in V^{\prime}$ and $v \in V$, then $F(v)=\langle F, v\rangle$. If $\mathbf{T}: V \longrightarrow W$ is a linear map from $V$ to the finite dimensional vector space $W$, then $\mathbf{T}^{t}: W^{\prime} \longrightarrow V^{\prime}$ is defined by

$$
\left\langle\mathbf{T}^{t} \alpha, v\right\rangle=\langle\alpha, \mathbf{T} v\rangle \quad \forall \alpha \in W^{\prime}, v \in V
$$

We will identify $V^{\prime \prime}$ (resp. $W^{\prime \prime}$ ) with $V$ (resp. $W$ ), and hence $\left(\mathbf{T}^{t}\right)^{t}$ is identified with $\mathbf{T}$. If $\mathbf{T}: V \longrightarrow V^{\prime}$ is a linear map, then we say that $\mathbf{T}$ is symmetric positive-definite if the following conditions are satisfied.

$$
\begin{array}{rlrl}
\left\langle\mathbf{T} v_{1}, v_{2}\right\rangle & =\left\langle\mathbf{T} v_{2}, v_{1}\right\rangle & & \forall v_{1}, v_{2} \in V, \\
\langle\mathbf{T} v, v\rangle>0 & & \forall v \in V, v \neq 0 .
\end{array}
$$

Following this convention, we can introduce the operators $\mathbf{S}: \mathcal{M}_{h}(\Gamma) \longrightarrow\left[\mathcal{M}_{h}(\Gamma)\right]^{\prime}$ and $\mathbf{S}_{j}: \mathcal{M}_{h}\left(\Gamma_{j}\right) \longrightarrow\left[\mathcal{M}_{h}\left(\Gamma_{j}\right)\right]^{\prime}$ as follows:

$$
\begin{aligned}
\langle\mathbf{S} v, w\rangle & =a_{h}(v, w) & \forall v, w \in \mathcal{M}_{h}(\Gamma), \\
\left\langle\mathbf{S}_{j} v, w\right\rangle & =a_{h, j}(v, w) & \forall v, w \in \mathcal{M}_{h}\left(\Gamma_{j}\right) .
\end{aligned}
$$

Note that $\mathbf{S}$ is symmetric positive-definite because of (1.6), but $\mathbf{S}_{j}$ is only symmetric positive semi-definite (i.e., the strict inequality in (2.6) is replaced by $\geq$ ) when $\partial \Omega_{j} \cap \partial \Omega=$ $\emptyset$. In general, the kernel (null space) of $\mathbf{S}_{j}$ is a subspace of $\mathcal{P}_{1}\left(\Omega_{j}\right)$. 
Let $v, w \in \mathcal{M}_{h}(\Gamma)$. We have, by splitting the integral in (1.4) over the subdomains,

$$
a_{h}(v, w)=\sum_{j=1}^{J} a_{h, j}\left(\mathbf{R}_{j} v, \mathbf{R}_{j} w\right)
$$

It follows from $(2.7)-(2.9)$ that

$$
\mathbf{S}=\sum_{j=1}^{J} \mathbf{R}_{j}^{t} \mathbf{S}_{j} \mathbf{R}_{j}
$$

As was mentioned in the introduction, the goal of a nonoverlapping domain decomposition method is to find a good preconditioner for $\mathbf{S}$. In view of (2.10) and (2.3), it is natural to use $\sum_{j=1}^{J} \mathbf{E}_{j} \mathbf{S}_{j}^{-1} \mathbf{E}_{j}^{t}$ as a preconditioner for $\mathbf{S}$. Unfortunately, the operator $\mathbf{S}_{j}$ is in general singular. This difficulty can be overcome by the BDD method.

Let $\dot{\mathcal{M}}_{h}\left(\Gamma_{j}\right)$ be the subspace of $\mathcal{M}_{h}\left(\Gamma_{j}\right)$ whose members vanish at the cross points of $\Omega_{j}$ and $\mathbf{J}_{j}: \dot{\mathcal{M}}_{h}\left(\Gamma_{j}\right) \longrightarrow \mathcal{M}_{h}\left(\Gamma_{j}\right)$ be the natural injection. Note that $a_{h, j}(\cdot, \cdot)$ restricted to $\dot{\mathcal{M}}_{h}\left(\Gamma_{j}\right)$ is symmetric positive-definite, since the only linear polynomial vanishing at all the cross points of $\Omega_{j}$ is the zero polynomial. The operator $\mathscr{S}_{j}: \dot{\mathcal{M}}_{h}\left(\Gamma_{j}\right) \longrightarrow\left[\dot{\mathcal{M}}_{h}\left(\Gamma_{j}\right)\right]^{\prime}$ defined by

$$
\left\langle\mathscr{S}_{j} v, w\right\rangle=a_{h, j}(v, w) \quad \forall v, w \in \dot{\mathcal{M}}_{h}\left(\Gamma_{j}\right)
$$

is therefore symmetric positive-definite, and $\mathscr{S}_{j}^{-1}$ can be used as part of a preconditioner for $\mathbf{S}$. However, information is being lost when we restrict to the space $\dot{\mathcal{M}}_{h}\left(\Gamma_{j}\right)$. This can be compensated for by collecting all the discarded functions into a "coarse grid space" which also provides global communication among the subdomains.

Let $\mathbf{D}_{j}: \mathcal{M}_{h}\left(\Gamma_{j}\right) \longrightarrow \mathcal{M}_{h}\left(\Gamma_{j}\right)$ be defined by (i) $\left(\partial\left(\mathbf{D}_{j} v\right) / \partial n\right)(m)=(1 / 2)(\partial v / \partial n)(m)$ at any midpoints $m$ of $\mathcal{T}_{h}$ along $\Gamma_{j}$, (ii) $\left(\mathbf{D}_{j} v\right)(p)=v(p) / 2$ at any vertex $p$ of $\mathcal{T}_{h}$ along $\Gamma_{j}$ that is not a cross point of $\Omega_{j}$, (iii) $\left(\mathbf{D}_{j} v\right)(p)=v(p) / n_{p}$ at a cross point $p$ of $\Omega_{j}$, where $n_{p}$ is the number of subdomains that have $p$ as a cross point. It is easy to see that the operators $\mathbf{D}_{j}$ form a partition of unity in the following sense:

$$
\sum_{j=1}^{J} \mathbf{E}_{j} \mathbf{D}_{j} \mathbf{R}_{j}=\mathbf{I d}
$$

where Id $: \mathcal{M}_{h}(\Gamma) \longrightarrow \mathcal{M}_{h}(\Gamma)$ is the identity operator.

Let $Z_{j}$ be the $a_{h, j}(\cdot, \cdot)$-orthogonal complement of $\dot{\mathcal{M}}_{h}\left(\Gamma_{j}\right)$ in $\mathcal{M}_{h}\left(\Gamma_{j}\right)$, i.e., $Z_{j}=\{v \in$ $\left.\mathcal{M}_{h}\left(\Gamma_{j}\right): a_{h, j}(v, w)=0 \quad \forall w \in \dot{\mathcal{M}}_{h}\left(\Gamma_{j}\right)\right\}$. (It is clear that $\operatorname{Ker} \mathbf{S}_{j} \subseteq Z_{j}$, and in general $\operatorname{Ker} \mathbf{S}_{j} \neq Z_{j}$. The details of the construction of $Z_{j}$ are given below in Remark 2.3.) We can now define the "coarse grid space" $\mathcal{M}_{H}$ to be

$$
\mathcal{M}_{H}=\sum_{j=1}^{J} \mathbf{E}_{j} \mathbf{D}_{j} Z_{j}
$$


which is a subspace of $\mathcal{M}_{h}(\Gamma)$. The natural injection of $\mathcal{M}_{H}$ into $\mathcal{M}_{h}(\Gamma)$ is denoted by $\mathbf{I}_{0}$, and $\mathbf{S}_{0}: \mathcal{M}_{H} \longrightarrow \mathcal{M}_{H}^{\prime}$ is defined by

$$
\left\langle\mathbf{S}_{0} v, w\right\rangle=a_{h}(v, w) \quad \forall v, w \in \mathcal{M}_{H} .
$$

Clearly, $\mathbf{S}_{0}$ is symmetric positive-definite.

Let $\mathbf{P}_{0}: \mathcal{M}_{h}(\Gamma) \longrightarrow \mathcal{M}_{h}(\Gamma)$ be the $a_{h}(\cdot, \cdot)$-orthogonal projection operator associated with $\mathcal{M}_{H}$, i.e.,

$$
\mathbf{P}_{0}=\mathbf{I}_{0} \mathbf{S}_{0}^{-1} \mathbf{I}_{0}^{t} \mathbf{S}
$$

Using the symmetry of $\mathbf{S}$ and $\mathbf{S}_{0}$, we have

$$
\mathbf{P}_{0}^{t}=\mathbf{S} \mathbf{I}_{0} \mathbf{S}_{0}^{-1} \mathbf{I}_{0}^{t}
$$

Let $\mathbf{I}_{j}: \dot{\mathcal{M}}_{h}\left(\Gamma_{j}\right) \longrightarrow \mathcal{M}_{h}(\Gamma)$ be defined by

$$
\mathbf{I}_{j}=\left(\mathbf{I} \mathbf{d}-\mathbf{P}_{0}\right) \mathbf{E}_{j} \mathbf{D}_{j} \mathbf{J}_{j},
$$

Then the BDD preconditioner $\mathbf{B}:\left[\mathcal{M}_{h}(\Gamma)\right]^{\prime} \rightarrow \mathcal{M}_{h}(\Gamma)$ for $\mathbf{S}$ is defined by (cf. [27], [28], $[25],[26],[17])$

$$
\mathbf{B}=\mathbf{I}_{0} \mathbf{S}_{0}^{-1} \mathbf{I}_{0}^{t}+\sum_{j=1}^{J} \mathbf{I}_{j} \mathscr{S}_{j}^{-1} \mathbf{I}_{j}^{t}
$$

Let $\alpha \in\left[\mathcal{M}_{h}(\Gamma)\right]^{\prime}$. Using (2.15) and (2.16) we can compute $v=\mathbf{B} \alpha$ as follows:

$$
\begin{array}{ll}
\text { Step } 1 & \text { Compute } w=\mathbf{I}_{0} \mathbf{S}_{0}^{-1} \mathbf{I}_{0}^{t} \alpha . \\
\text { Step } 2 & \text { Compute } \beta=\alpha-\mathbf{S} w . \\
\text { Step } 3 & \text { Set } \beta_{j}=\mathbf{D}_{j}^{t} \mathbf{E}_{j}^{t} \beta \text { for } 1 \leq j \leq J \\
\text { Step } 4 & \text { Compute } v_{j}=\mathbf{J}_{j} \mathscr{S}_{j}^{-1} \mathbf{J}_{j}^{t} \beta_{j} \text { for } 1 \leq j \leq J \\
\text { Step } 5 & \text { Set } \hat{v}=\sum_{j=1}^{J} \mathbf{E}_{j} \mathbf{D}_{j} v_{j} . \\
\text { Step } 6 & \text { Compute } \gamma=\alpha-\mathbf{S} \hat{v} . \\
\text { Step } 7 & \text { Compute } v_{0}=\mathbf{I}_{0} \mathbf{S}_{0}^{-1} \mathbf{I}_{0}^{t} \gamma .
\end{array}
$$

Then

$$
v=v_{0}+\hat{v}
$$

Remark 2.1. The execution of $\mathbf{S}$ in step 2 and step 6 is equivalent to solving plate bending problems on the $\Omega_{j}$ 's with essential (Dirichlet) boundary condition on the $\Gamma_{j}$ 's, and the execution of $\mathscr{S}_{j}^{-1}$ in step 4 is equivalent to solving plate bending problems on the $\Omega_{j}$ 's with natural (Neumann) boundary condition on the $\Gamma_{j}$ 's. They can both be done in parallel.

Remark 2.2. Note that the $\beta$ computed by Steps 1 and 2 is just $\left(\mathbf{I d}-\mathbf{P}_{0}\right)^{t} \alpha$ and therefore

$$
\langle\beta, v\rangle=0 \quad \forall v \in \mathcal{M}_{H}
$$


It follows from (2.13) and (2.19) that the $\beta_{j}$ in Step 3 satisfies

$$
\left\langle\beta_{j}, z_{j}\right\rangle=0 \quad \forall z_{j} \in Z_{j}
$$

which is the solvability condition of the equation

$$
\mathbf{S}_{j} \hat{v}_{j}=\beta_{j}
$$

The $v_{j}$ in Step 4 is the solution of $(2.21)$ in $\dot{\mathcal{M}}_{h}\left(\Gamma_{j}\right)$. Since Steps $5-7$ amount to the computation of $\mathbf{I}_{0} \mathbf{S}_{0}^{-1} \mathbf{I}_{0}^{t} \alpha+\sum_{j=1}^{J}\left(\mathbf{I d}-\mathbf{P}_{0}\right) \mathbf{E}_{j} \mathbf{D}_{j} v_{j}$, the final output $v$ of the algorithm is not affected by replacing $v_{j}$ with any other solutions of $(2.21)$. Therefore, Step 4 can be replaced by Step $4^{\prime}$ Find $v_{j} \in \mathcal{M}_{h}\left(\Gamma_{j}\right)$ such that $\mathbf{S}_{j} v_{j}=\beta_{j}$.

Remark 2.3. Let $C_{j}$ be the set of cross points of $\Omega_{j}$ that are not on $\partial \Omega$. For each $p \in C_{j}$, we introduce the subspace $V_{p}=\left\{v \in \mathcal{M}_{h}\left(\Gamma_{j}\right): v(q)=0 \forall q \in C_{j} \backslash\{p\}\right\}$, and determine a function $z_{p} \in V_{p}$ as follows.

Case 1: $\operatorname{dim}\left(\mathcal{P}_{1}\left(\Omega_{j}\right) \cap V_{p}\right)=1$.

In this case we take $z_{p}$ to be any basis function of $\mathcal{P}_{1}\left(\Omega_{j}\right) \cap V_{p}$.

Case 2: $\operatorname{dim}\left(\mathcal{P}_{1}\left(\Omega_{j}\right) \cap V_{p}\right)=0$.

In this case we take $z_{p} \in V_{p}$ to be the function that satisfies

$$
a_{h, j}\left(z_{p}, v\right)=v(p) \quad \forall v \in V_{p}
$$

The subspace $Z_{j}$ is spanned by the functions $z_{p}$ where $p$ runs through all the cross points in $C_{j}$.

Remark 2.4. The singularity of $\mathbf{S}_{j}$ can also be overcome by factoring out its kernel. We can define a preconditioner $\mathbf{K}$ by replacing $\dot{\mathcal{M}}_{h}\left(\Gamma_{j}\right)$ with any subspace that is complementary to the kernel of $\mathbf{S}_{j}$, and replacing $Z_{j}$ with $\operatorname{Ker} \mathbf{S}_{j}$ in the construction of $\mathcal{M}_{H}$. This is in fact the BDD preconditioner for second order problems. However, as pointed out by Le Tallec, Mandel and Vidrascu (cf. [25]), the condition number of KS will grow at the rate of $(H / h)^{2}$ for fourth order problems (cf. Remark 6.4). The suppression of the nodal values at the cross points is crucial for the condition number estimate $C[1+\ln (H / h)]^{2}$ in the case of fourth order problems.

The preconditioner B defined by (2.18) has the form of an additive Schwarz preconditioner. The operator $\mathbf{B S}$ is symmetric with respect to the bilinear form $\langle\mathbf{S} \cdot, \cdot\rangle$, and the condition number of BS can be estimated by the following lemma (cf. [19], [23], [31], [32], $[38]$ and [36]).

Lemma 2.5. Let $C_{1}$ and $C_{2}$ be two positive constants such that

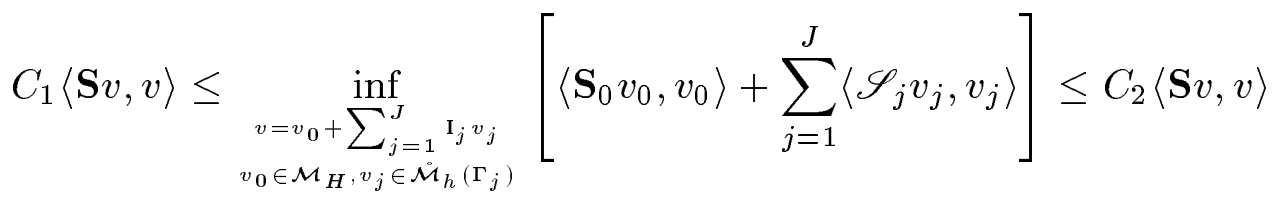


for all $v \in \mathcal{M}_{h}(\Gamma)$. Then we have the following estimates on the eigenvalues of BS:

$$
\lambda_{\max }(\mathbf{B S}) \leq C_{1}^{-1} \quad \text { and } \quad \lambda_{\min }(\mathbf{B S}) \geq C_{2}^{-1} .
$$

Moreover, if $C_{1}$ (resp. $C_{2}$ ) is the largest (resp. smallest) constant for which (2.23) holds, then the inequalities in (2.24) become identities.

It turns out that the second inequality in (2.23) holds for $C_{2}=1$. The analysis of the BDD preconditioner therefore consists mainly of finding a constant $C_{1}$ that satisfies the first inequality in (2.23). The condition number estimate for $\mathbf{B S}$ will be given in Section 6 after the preparations in the next three sections.

\section{Conforming Relatives}

Let $T$ be a triangle. The space of shape functions for the Hsieh-Clough-Tocher (HCT) macro element (cf. [15]) is $\mathcal{H C} \mathcal{T}(T)=\left\{v \in C^{1}(T): v\right.$ is piecewise cubic on the three triangles formed by connecting the vertices of $T$ to its centroid\}. A function $v$ in $\mathcal{H C T}(T)$ is completely determined by the values of $v$ and $\nabla v$ at the vertices and $\partial v / \partial n$ at the three midpoints (cf. Figure 3). The HCT finite element space associated with the triangulation $\mathcal{T}_{h}$ is a subspace of $H^{2}(\Omega)$, i.e., it is conforming for the plate bending problem.

The HCT finite element is a relative of the Morley finite element in the sense that the space of shape functions of the Morley finite element, namely $\mathcal{P}_{2}(T)$, is a subspace of $\mathcal{H C} \mathcal{T}(T)$ and the nodal variables of the Morley element are also nodal variables of the HCT element (cf. Figure 2 and Figure 3).

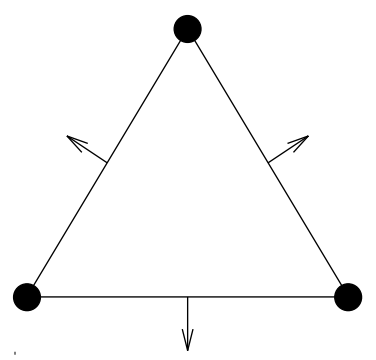

Figure 2

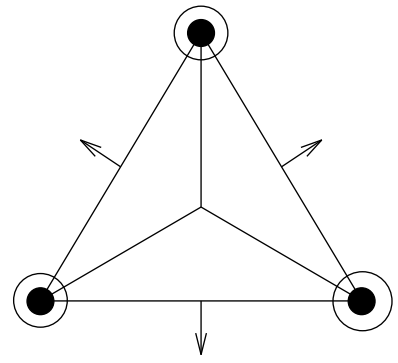

Figure 3

Let $D$ be a polygonal domain with a triangulation $\mathscr{T}_{h}$, and $\mathcal{M}_{h}(\bar{D})$ and $\mathcal{H C} \mathcal{T}_{h}(\bar{D})$ be the Morley and Hsieh-Clough-Tocher finite element spaces associated with $\mathscr{T}_{h}$, without boundary conditions. The following constructions relate these two finite element spaces.

Let $v \in \mathcal{M}_{h}(\bar{D})$. Observe that

$$
q^{\prime}((a+b) / 2)=\frac{q(b)-q(a)}{b-a} \quad \text { for any quadratic polynomial } q .
$$

Hence the gradient $\nabla v$ is well-defined at all of the midpoints. We can therefore define $\tilde{v} \in \mathcal{H C}_{h}(\bar{D})$ as follows:

$$
\begin{aligned}
\tilde{v}(p) & =v(p) & & \forall \text { vertices } p \\
\left(\frac{\partial \tilde{v}}{\partial n}\right)(m) & =\left(\frac{\partial v}{\partial n}\right)(m) & & \forall \text { midpoints } m \\
(\nabla \tilde{v})(p) & =(\nabla v)\left(m_{p}\right) & & \forall \text { vertices } p, \text { where } m_{p} \text { is an adjacent midpoint. }
\end{aligned}
$$


We say that $m_{p}$ is an adjacent midpoint of the vertex $p$ if $m_{p}$ and $p$ belong to the same edge in $\mathscr{T}_{h}$. The choice of $m_{p}$ is of course not unique. This fact can be used later to our advantage.

Conversely, given $w \in \mathcal{H C} \mathcal{T}_{h}(\bar{D})$, we can define $\hat{w} \in \mathcal{M}_{h}(\bar{D})$ by

$$
\begin{array}{rlrl}
\hat{w}(p) & =w(p) & & \forall \text { vertices } p \\
\frac{\partial \hat{w}}{\partial n}(m) & =\frac{\partial w}{\partial n}(m) & \forall \text { midpoints } m .
\end{array}
$$

We can compare $v$ and $\tilde{v}(w$ and $\hat{w})$ in terms of conforming and nonconforming Sobolev semi-norms. For any open set $G$ the Sobolev $H^{2}$ semi-norm is defined by

$$
|v|_{H^{2}(G)}=\left(\int_{G} \sum_{i, j=1}^{2}\left(v_{x_{i} x_{j}}\right)^{2} d x\right)^{1 / 2} \quad \forall v \in H^{2}(G)
$$

and the nonconforming $H^{2}$-semi-norm associated with $\mathscr{T}_{h}$ is defined by

$$
|v|_{H^{2}\left(\mathscr{T}_{h}\right)}=\left(\sum_{T \in \mathscr{T}_{h}}|v|_{H^{2}(T)}^{2}\right)^{1 / 2}
$$

for all functions $v$ such that $\left.v\right|_{T} \in H^{2}(T)$ for all $T \in \mathscr{T}_{h}$.

In order to avoid the proliferation of constants, we will use the notation $F \lesssim G$ to represent the statement that $F$ is bounded by $G$ multiplied by a constant which only depends on the minimum angle of $\mathscr{T}_{h}$. The statement $F \approx G$ means $F \lesssim G$ and $G \lesssim F$.

Let $T \in \mathscr{T}_{h}$. By a scaling argument, we have

$$
\|v\|_{L^{2}(T)}^{2} \approx(\operatorname{diam} T)^{2} \sum_{j=1}^{3}\left[v\left(p_{j}\right)\right]^{2}+(\operatorname{diam} T)^{4} \sum_{j=1}^{3}\left\{\left[\frac{\partial v}{\partial n}\left(m_{j}\right)\right]^{2}+\left|(\nabla v)\left(p_{j}\right)\right|^{2}\right\}
$$

for all $v \in \mathcal{H C} \mathcal{T}(T)$, where $p_{1}, p_{2}$ and $p_{3}$ are the vertices of $T$, and $m_{1}, m_{2}, m_{3}$ are the midpoints of the edges of $T$.

We can now compare $v$ with $\tilde{v}$ and $w$ with $\hat{w}$.

Lemma 3.1. Let $v \in \mathcal{M}_{h}(\bar{D})$ and $\tilde{v} \in \mathcal{H C} \mathcal{T}_{h}(\bar{D})$ be defined by (3.2). Then we have

$$
|\tilde{v}|_{H^{2}(D)} \lesssim|v|_{H^{2}\left(\mathcal{T}_{h}\right)}
$$

Proof. Let $T_{*} \in \mathscr{T}_{h}$ and $m_{1}, m_{2}$ be two distinct midpoints of $T_{*}$. Note that on the finitedimensional space $\mathcal{P}_{2}\left(T_{*}\right) / \mathcal{P}_{1}\left(T_{*}\right)$, the formula $\left|(\nabla q)\left(m_{1}\right)-(\nabla q)\left(m_{2}\right)\right|$ defines a semi-norm, while $|q|_{H^{2}\left(T_{*}\right)}$ defines a norm. Hence, by a scaling argument, we have

$$
\left|(\nabla q)\left(m_{1}\right)-(\nabla q)\left(m_{2}\right)\right| \lesssim|q|_{H^{2}\left(T_{*}\right)} \quad \forall q \in \mathcal{P}_{2}\left(T_{*}\right)
$$


Let $v_{*} \in \mathcal{H C} \mathcal{T}\left(T_{*}\right)$ be related to $\left.v\right|_{T_{*}}$ by (3.2), where $\nabla v^{*}$ at a vertex $p$ of $T$ equals $\nabla v$ at that midpoint of $T$ which precedes $p$ in the counterclockwise sense. Using a standard inverse estimate (cf. [14], [13]), (3.2c), (3.6) and (3.8), we obtain

$$
\begin{aligned}
|v-\tilde{v}|_{H^{2}\left(T_{*}\right)}^{2} & \lesssim(\operatorname{diam} T)^{-4}\|v-\tilde{v}\|_{L^{2}\left(T_{*}\right)}^{2} \\
& \lesssim(\operatorname{diam} T)^{-4}\left\|v-v_{*}\right\|_{L^{2}\left(T_{*}\right)}^{2}+\sum_{T \in S\left(T_{*}\right)}|v|_{H^{2}(T)}^{2},
\end{aligned}
$$

where

$$
S\left(T_{*}\right)=\text { the union of all } T \in \mathscr{T}_{h} \text { such that } \bar{T} \cap \bar{T}_{*} \neq \emptyset \text {. }
$$

Since $\left\|v-v^{*}\right\|_{L^{2}\left(T_{*}\right)}$ defines a semi-norm on the finite-dimensional space $\mathcal{P}_{2}\left(T_{*}\right) / \mathcal{P}_{1}\left(T_{*}\right)$, we again obtain by a scaling argument that

$$
\left\|v-v^{*}\right\|_{L^{2}\left(T_{*}\right)} \lesssim \operatorname{diam}(T)^{2}|v|_{H^{2}(T)} .
$$

The estimate (3.7) follows from (3.9) and (3.11).

A similar but simpler argument gives the next lemma.

Lemma 3.2. Let $w \in \mathcal{H C T}_{h}(\bar{D})$ and $\hat{w} \in \mathcal{M}_{h}(\bar{D})$ be defined by (3.3). Then we have

$$
|\hat{w}|_{H^{2}\left(\mathscr{T}_{h}\right)} \lesssim|w|_{H^{2}(D)} .
$$

Combining (3.7), (3.12) and the identity $\hat{\tilde{v}}=v$, we have the following corollary.

Corollary 3.3. Let $v \in \mathcal{M}_{h}(\bar{D})$ and $\tilde{v} \in \mathcal{H C} \mathcal{T}_{h}(\bar{D})$ be defined by (3.2). Then we have

$$
|\tilde{v}|_{H^{2}(D)} \approx|v|_{H^{2}\left(\mathscr{T}_{h}\right)} .
$$

Finally we describe the construction of an $\mathcal{H C} \mathcal{T}$ interpolant $v_{\zeta}$ for a function $\zeta \in$ $H^{2}(D)$. We assume that a unit normal $n_{e}$ has been chosen for each edge $e \in \mathscr{T}_{h}$ and define $\zeta_{e, 3} \in \mathcal{P}_{3}(e)$ and $\zeta_{e, 2} \in \mathcal{P}_{2}(e)$ to be the $L^{2}(e)$ projection of $\left.\zeta\right|_{e}$ and $\left.\left(\partial \zeta / \partial n_{e}\right)\right|_{e}$ respectively. We will assign the nodal values of $v_{\zeta} \in \mathcal{H C}_{h}(\bar{D})$ as follows. Let $m$ be a midpoint of an edge $e$ in $\mathscr{T}_{h}$. We define

$$
\left(\frac{\partial v_{\zeta}}{\partial n}\right)(m)=\zeta_{e, 2}(m)
$$

Let $p$ be a vertex in $\mathscr{T}_{h}$ and $e$ be an edge in $\mathscr{T}_{h}$ with $p$ as an endpoint. We define

$$
v_{\zeta}(p)=\zeta_{e, 3}(p),
$$

and $\left(\nabla v_{\zeta}\right)(p)$ to be the vector satisfying

$$
\begin{aligned}
\left(\nabla v_{\zeta}\right)(p) \cdot n_{e} & =\zeta_{e, 2}(p), \\
\left(\nabla v_{\zeta}\right)(p) \cdot t_{e} & =\zeta_{e, 3}^{\prime}(p),
\end{aligned}
$$


where $t_{e}$ is a unit tangent vector of $e$ and $\zeta_{e, 3}^{\prime}$ is the derivative of $\zeta_{e, 3}$ in the direction of $t_{e}$. Note that the choice of the nodal values of $v_{\zeta}$ at a vertex $p$ is not unique, since there are many edges emanating from $p$.

Lemma 3.4. It holds that

$$
\left|v_{\zeta}\right|_{H^{2}(D)} \lesssim|\zeta|_{H^{2}(D)}
$$

Proof. The following arguments are similar to those in the proof of Lemma 3.1.

Let $e_{1}$ and $e_{2}$ be two sides of $T_{*} \in \mathscr{T}_{h}$ and $p$ be the common vertex of $e_{1}$ and $e_{2}$. Suppose $v_{\zeta, e_{1}}(p)$ and $\left(\nabla v_{\zeta, e_{1}}\right)(p)$ are defined by (3.15)-(3.17) using $\zeta_{e_{1}, 3}$ and $\zeta_{e_{1}, 2}$, and $v_{\zeta, e_{2}}(p)$ and $\left(\nabla v_{\zeta, e_{2}}\right)(p)$ are defined by $(3.15)-(3.17)$ using $\zeta_{e_{2}, 3}$ and $\zeta_{e_{2}, 2}$. It is clear that $v_{\zeta, e_{1}}(p)=v_{\zeta, e_{2}}(p)$ and $\left(\nabla v_{\zeta, e_{1}}\right)(p)=\left(\nabla v_{\zeta, e_{2}}\right)(p)$ when $\zeta \in \mathcal{P}_{1}\left(T_{*}\right)$. Therefore, by the Bramble-Hilbert lemma (cf. [7]) and a scaling argument, we have

$$
\begin{aligned}
\left|v_{\zeta, e_{1}}(p)-v_{\zeta, e_{2}}(p)\right| & \lesssim\left(\operatorname{diam} T_{*}\right)|\zeta|_{H^{2}\left(T_{*}\right)} \\
\left|\left(\nabla v_{\zeta, e_{1}}\right)(p)-\left(\nabla v_{\zeta, e_{2}}\right)(p)\right| & \lesssim|\zeta|_{H^{2}\left(T_{*}\right)} .
\end{aligned}
$$

Let $v_{\zeta_{T_{*}}} \in \mathcal{H C} \mathcal{T}\left(T_{*}\right)$ be defined by $(3.14)-(3.17)$, where the nodal values of $v_{\zeta_{T_{*}}}$ at a vertex $p$ of $T_{*}$ are defined by using $\zeta_{e, 3}$ and $\zeta_{e, 2}$, with $e$ being the edge of $T_{*}$ preceding $p$ in the counterclockwise sense.

By (3.14)-(3.17), (3.19)-(3.20), (3.6) and a standard inverse estimate, we have

$$
\begin{aligned}
\left|v_{\zeta}\right|_{H^{2}\left(T_{*}\right)} & \lesssim\left|v_{\zeta,_{*}}\right|_{H^{2}\left(T_{*}\right)}+\left(\operatorname{diam} T_{*}\right)^{-2}\left|v_{\zeta}-v_{\zeta, T_{*}}\right|_{L^{2}\left(T_{*}\right)} \\
& \lesssim\left|v_{\zeta, T_{*}}\right|_{H^{2}\left(T_{*}\right)}+|\zeta|_{H^{2}\left(S\left(T_{*}\right)\right)},
\end{aligned}
$$

where $S\left(T_{*}\right)$ is defined in (3.10). On the other hand, a scaling argument yields

$$
\left|v_{\zeta, T_{*}}\right| \lesssim|\zeta|_{H^{2}\left(T_{*}\right)}
$$

The estimate (3.18) follows from (3.21) and (3.22).

Remark 3.5. The construction of $v_{\zeta}$ is motivated by the idea of Scott and Zhang in [33]. It can be shown that

$$
\left|\zeta-v_{\zeta}\right|_{H^{k}(D)} \lesssim h^{2-k}|\zeta|_{H^{2}(D)} \quad \text { for } \quad k=0,1
$$

But (3.18) is all that we need later.

Remark 3.6. Conforming relatives exist for many other nonconforming plate elements. For example, a conforming relative for the Zienkiewicz element (cf. [3]) (resp., the Fraeijs de Veubeke element (cf. [22]), the Adini element (cf. [1]) and the incomplete biquadratic element (cf. [34])) is the Bell element (cf. [4]) (resp., the sixth degree Argyris element (cf. [2]), the Bogner-Fox-Schmit element (cf. [6]) and the Fraeijs de Veubeke-Sander element (cf. [21])). 


\section{A Norm Equivalence}

One of the key ingredients in the condition number estimate for nonoverlapping domain decomposition methods is the equivalence of the energy norm and a certain fractionalorder Sobolev trace norm on the space of discrete harmonic functions ( $2^{\text {nd }}$ order problems) or discrete biharmonic functions ( $4^{\text {th }}$ order problems). We will show in this section how to modify this norm equivalence for the nonconforming Morley finite element. Our construction is based on the concept of conforming relatives developed in the previous section.

We first state the precise conditions on the shape regularity of the subdomains $\Omega_{j}$. Recall that $H=\max _{1 \leq j \leq J} \operatorname{diam} \Omega_{j}$. We assume that there exist reference domains $D_{k}$, $1 \leq k \leq K$, with the following properties:

$$
\operatorname{diam} D_{k}=1
$$

and for each $\Omega_{j}$, there exists a $C^{2}$ diffeomorphism $T_{k, j}$ from $\bar{D}_{k}$ to $\bar{\Omega}_{j}$ such that

The absolute values of the $\ell^{\text {th }}$ order derivatives of $T_{k, j}\left(\operatorname{resp} . T_{k, j}^{-1}\right.$ ) are $\lesssim H^{\ell}$ (resp. $\left.H^{-\ell}\right)$ for $\ell=0,1,2$.

(Here and in Section 6 the constant in the statement $F \lesssim G$ is independent of $h, H$ and $J$.) Note that (4.1) and (4.2) imply in particular that diam $\Omega_{j} \approx H$ for $1 \leq j \leq J$.

We also need a fractional-order Sobolev semi-norm. Let $D$ be a bounded polygonal domain. The semi-norm $|\cdot|_{H^{1 / 2}(\partial D)}$ is defined by (cf. [30])

$$
|v|_{H^{1 / 2}(\partial D)}=\left(\int_{\partial D} \int_{\partial D} \frac{|v(x)-v(y)|^{2}}{|x-y|^{2}} d s(x) d s(y)\right)^{1 / 2} \quad \forall v \in H^{1 / 2}(\partial D),
$$

where $d s$ is the arc-length differential.

Throughout this section we use $C_{D}$ to denote a generic positive constant which only depends on $D$.

Lemma 4.1. There exists a constant $C_{D}$ such that

$$
|\nabla w|_{H^{1 / 2}(\partial D)} \leq C_{D}|w|_{H^{2}(D)} \quad \forall w \in H^{2}(D)
$$

Proof. By the trace theorem (cf. [24]) we have

$$
|\nabla w|_{H^{1 / 2}(\partial D)}=|\nabla(w+g)|_{H^{1 / 2}(\partial D)} \leq C_{D}\|w+g\|_{H^{2}(D)} \quad \forall g \in \mathcal{P}_{1}(D)
$$

The estimate (4.4) follows from (4.5) and the Bramble-Hilbert lemma (cf. [7]).

Since the natural injection from $H^{1 / 2}(\partial D)$ into $L^{2}(\partial D)$ is a compact operator (cf. [30]), the usual norm equivalence argument for Sobolev spaces (cf. [30]) yields

$$
\|w\|_{L^{2}(\partial D)} \leq C_{D}\left(\left|\int_{\partial D} w d s\right|+|w|_{H^{1 / 2}(D)}\right) \quad \forall w \in H^{1 / 2}(\partial D) .
$$


The next lemma follows from (4.6).

Lemma 4.2. There exists a constant $C_{D}$ such that

$$
\|w\|_{L^{2}(\partial D)} \leq C_{D}|w|_{H^{1 / 2}(\partial D)}
$$

for all $w \in H^{1 / 2}(\partial D)$ such that $\int_{\partial D} w d s=0$.

Lemma 4.3. There exists a constant $C_{D}$ such that given any $w \in H^{2}(D)$, we can find a function $\tilde{w} \in H^{2}(D)$ that satisfies

$$
\begin{aligned}
& \left.\tilde{w}\right|_{\partial D}=\left.w\right|_{\partial D} \text { and }\left.\nabla \tilde{w}\right|_{\partial D}=\left.\nabla w\right|_{\partial D}, \\
& |\tilde{w}|_{H^{2}(D)} \leq C_{D}|\nabla w|_{H^{1 / 2}(\partial D)}
\end{aligned}
$$

Proof. Let the constants $a, b$ and $c$ be defined by

$$
\int_{\partial D}\left[\left(\partial w / \partial x_{1}\right)-a\right] d s=\int_{\partial D}\left[\left(\partial w / \partial x_{2}\right)-b\right] d s=\int_{\partial D}\left[w-\left(a x_{1}+b x_{2}+c\right)\right] d s=0
$$

Let $g=a x_{1}+b x_{2}+c$. We have, by (4.10),

$$
\int_{\partial D}(w-g) d s=\int_{\partial D}\left(\partial(w-g) / \partial x_{1}\right) d s=\int_{\partial D}\left(\partial(w-g) / \partial x_{2}\right) d s=0
$$

It follows from Lemma 4.2 that

$$
\|w-g\|_{L^{2}(\partial D)}+\|\nabla(w-g)\|_{L^{2}(\partial D)} \leq C_{D}|\nabla(w-g)|_{H^{1 / 2}(\partial D)}=C_{D}|\nabla w|_{H^{1 / 2}(\partial D)}
$$

There exists by the trace theorem (cf. [24]) a function $w_{*} \in H^{2}(D)$ such that

$$
\begin{aligned}
& \left.w_{*}\right|_{\partial D}=\left.(w-g)\right|_{\partial D} \quad \text { and }\left.\quad \nabla w_{*}\right|_{\partial D}=\left.\nabla(w-g)\right|_{\partial D} \\
& \left|w_{*}\right|_{H^{2}(D)} \leq C_{D}\left(\|w-g\|_{L^{2}(\partial D)}+\|\nabla(w-g)\|_{L^{2}(\partial D)}+|\nabla(w-g)|_{H^{1 / 2}(\partial D)}\right) .
\end{aligned}
$$

Let $\tilde{w}=w_{*}+g$. Then $\tilde{w} \in H^{2}(D)$ and (4.8)-(4.9) follow from (4.12)-(4.14).

We now turn to the construction of the norm equivalence for the space $\mathcal{M}_{h}\left(\Gamma_{j}\right)$. Let $v \in \mathcal{M}_{h}\left(\Gamma_{j}\right)$ and $\tilde{v} \in \mathcal{H C} \mathcal{T}\left(\bar{\Omega}_{j}\right)$ be constructed by $(3.2)$. The functions $\mathscr{D}_{1} v=\left.\left(\partial \tilde{v} / \partial x_{1}\right)\right|_{\partial \Omega_{j}}$ and $\mathscr{D}_{2} v=\left.\left(\partial \tilde{v} / \partial x_{2}\right)\right|_{\partial \Omega_{j}}$ are piecewise quadratic with respect to the triangulation of $\partial \Omega_{j}$ induced by $\mathcal{T}_{h}$ and are continuous on $\partial \Omega_{j}$.

Definition 4.4. We say that $\left(\mathscr{D}_{1} v, \mathscr{D}_{2} v\right)$ is an $\mathcal{H C} \mathcal{T}$ trace of $\nabla v$.

Remark 4.5. $\left(\mathscr{D}_{1} v, \mathscr{D}_{2} v\right)$ can be constructed directly using only the nodal values of $v$ along $\partial \Omega_{j}$ and formula (3.2).

We can now prove the result on equivalent norms of the discrete biharmonic Morley functions. 
Lemma 4.6. Let $v \in \mathcal{M}_{h}\left(\Gamma_{j}\right)$ and $\left(\mathscr{D}_{1} v, \mathscr{D}_{2} v\right)$ be an $\mathcal{H C} \mathcal{T}$ conforming trace of $\nabla v$ Then we have

$$
\left|\mathscr{D}_{1} v\right|_{H^{1 / 2}\left(\partial \Omega_{j}\right)}+\left|\mathscr{D}_{2} v\right|_{H^{1 / 2}\left(\partial \Omega_{j}\right)} \approx|v|_{H^{2}\left(\mathcal{T}_{h}\right)}
$$

where the nonconforming semi-norm $|\cdot|_{H^{2}\left(\mathcal{T}_{h}\right)}$ with respect to the triangulation $\mathcal{T}_{h}$ is defined as in (3.5).

Proof. Observe first that, by (1.5) and (1.6) applied to $\Omega_{j}$, we have

$$
a_{h, j}(\cdot, \cdot) \approx|\cdot|_{H^{2}\left(\mathcal{T}_{h}\right)}^{2} \quad \text { on } \mathcal{M}_{h}\left(\Gamma_{j}\right)
$$

Let $\tilde{v} \in \mathcal{H C} \mathcal{T}\left(\bar{\Omega}_{j}\right)$ be constructed by (3.2) so that

$$
\left(\mathscr{D}_{1} v, \mathscr{D}_{2} v\right)=\left(\left.\left(\partial \tilde{v} / \partial x_{1}\right)\right|_{\partial \Omega_{j}},\left.\left(\partial \tilde{v} / \partial x_{2}\right)\right|_{\partial \Omega_{j}}\right)
$$

It follows from (4.17), Lemma 4.1 (for the reference domains) and a scaling argument using (4.1)-(4.2) that

$$
\left|\partial \tilde{v} / \partial x_{1}\right|_{H^{1 / 2}\left(\partial \Omega_{j}\right)}+\left|\partial \tilde{v} / \partial x_{2}\right|_{H^{1 / 2}\left(\partial \Omega_{j}\right)} \lesssim|\tilde{v}|_{H^{2}\left(\Omega_{j}\right)}
$$

Combining (4.17)-(4.18) and Corollary 3.3 we have

$$
\left|\mathscr{D}_{1} v\right|_{H^{1 / 2}\left(\partial \Omega_{j}\right)}+\left|\mathscr{D}_{2} v\right|_{H^{1 / 2}\left(\partial \Omega_{j}\right)} \lesssim|v|_{H^{2}\left(\mathcal{T}_{h}\right)}
$$

On the other hand, there exists by Lemma 4.3 (for the reference domains) and a scaling argument using (4.1)-(4.2), a function $\zeta \in H^{2}(D)$ such that

$$
\begin{aligned}
& \left.\zeta\right|_{\partial \Omega_{j}}=\left.\tilde{v}\right|_{\partial \Omega_{j}} \text { and }\left.\nabla \zeta\right|_{\partial \Omega_{j}}=\left.\nabla \tilde{v}\right|_{\partial \Omega_{j}}, \\
& |\zeta|_{H^{2}\left(\Omega_{j}\right)} \lesssim|\nabla \tilde{v}|_{H^{1 / 2}\left(\partial \Omega_{j}\right)}
\end{aligned}
$$

We can choose an $\mathcal{H C} \mathcal{T}$ interpolant $v_{\zeta}$ of $\zeta$ (cf. Section 3 ) such that the nodal values of $v_{\zeta}$ at a vertex $p$ on $\partial \Omega_{j}$ are defined using an edge $e$ on $\partial \Omega_{j}$ which has $p$ as an endpoint. Note that (4.20) implies $\left.\zeta\right|_{e} \in \mathcal{P}_{3}(e)$ and $\left.(\partial \zeta / \partial n)\right|_{e} \in \mathcal{P}_{2}(e)$ for any edge $e$ on $\partial \Omega_{j}$. Therefore it follows from (3.14)-(3.17) that $\left.v_{\zeta}\right|_{\partial \Omega_{j}}=\left.\zeta\right|_{\partial \Omega_{j}}$ and $\left.\nabla v_{\zeta}\right|_{\partial \Omega_{j}}=\left.\nabla \zeta\right|_{\partial \Omega_{j}}$.

By (3.18) and (4.20)-(4.21), the function $w=v_{\zeta} \in \mathcal{H C} \mathcal{T}\left(\bar{\Omega}_{j}\right)$ satisfies

$$
\begin{aligned}
& \left.w\right|_{\partial \Omega_{j}}=\left.\tilde{v}\right|_{\partial \Omega_{j}} \text { and }\left.\nabla w\right|_{\partial \Omega_{j}}=\left.\nabla \tilde{v}\right|_{\partial \Omega_{j}}, \\
& |w|_{H^{2}\left(\Omega_{j}\right)} \lesssim|\nabla \tilde{v}|_{H^{1 / 2}\left(\partial \Omega_{j}\right)} .
\end{aligned}
$$

Let $\hat{w} \in \mathcal{M}_{h}\left(\bar{\Omega}_{j}\right)$ be constructed from $w$ using (3.3). Then $\hat{w}$ shares the same nodal values as $v$ on $\partial \Omega_{j}$ by (4.22) and (3.2)-(3.3). It follows from (1.9), (3.12) and (4.16) that

$$
|v|_{H^{2}\left(\mathcal{T}_{h}\right)} \lesssim|\hat{w}|_{H^{2}\left(\mathcal{T}_{h}\right)} \lesssim|w|_{H^{2}\left(\Omega_{j}\right)}
$$


Combining (4.17), (4.23) and (4.24) we have

$$
|v|_{H^{2}\left(\mathcal{T}_{h}\right)} \lesssim\left|\mathscr{D}_{1} v\right|_{H^{1 / 2}\left(\partial \Omega_{j}\right)}+\left|\mathscr{D}_{2} v\right|_{H^{1 / 2}\left(\partial \Omega_{j}\right)} .
$$

Remark 4.7. The construction of $w$ in the proof of Lemma 4.6 is based on the ideas in the well-known extension theorems of linear elements (cf. [5] and [37]).

Finally we prove a lemma that will justify the extra effort involved in the construction of the coarse grid space $\mathcal{M}_{H}$ (cf. Remark 2.4.).

Lemma 4.8. Let $w \in H^{2}\left(\Omega_{j}\right)$ vanish at all of the vertices of $\Omega_{j}$. Then we have

$$
H^{-\frac{1}{2}}\|w\|_{L^{2}\left(\partial \Omega_{j}\right)}+H^{\frac{1}{2}}\|\nabla w\|_{L^{2}\left(\partial \Omega_{j}\right)} \lesssim H|\nabla w|_{H^{1 / 2}\left(\partial \Omega_{j}\right)} .
$$

Proof. It suffices to prove (4.26) on a reference domain $D$ for $H=1$. There exists by Lemma 4.5 a function $\tilde{w} \in H^{2}(D)$ which satisfies (4.8)-(4.9). Note that (4.8) implies that $\tilde{w}$ also vanishes at all the vertices of $D$. By the trace theorem we have

$$
\|w\|_{L^{2}(\partial D)}+\|\nabla w\|_{L^{2}(\partial D)} \leq C_{D}\|\tilde{w}\|_{H^{2}(D)} .
$$

Since the natural injection of $H^{2}(D)$ into $H^{1}(D)$ is compact, it follows from the usual norm equivalence argument for Sobolev spaces (cf. [30]) that

$$
\|v\|_{H^{2}(D)} \leq C_{D}\left(|v|_{H^{2}(D)}+\sum_{j=1}^{3}\left|v\left(p_{j}\right)\right|\right) \quad \forall v \in H^{2}(D),
$$

where $p_{1}, p_{2}$ and $p_{3}$ are three non-collinear vertices of $D$.

The estimate (4.26) for $D$ follows from (4.9), (4.27) and (4.28) (applied to $\tilde{w}$ ).

\section{An Extension Estimate}

We have shown in the previous section that the nonconforming energy norm of a discrete biharmonic Morley function $v$ on $\Omega_{j}$ is equivalent to the $|\cdot|_{H^{1 / 2}\left(\partial \Omega_{j}\right)}$ semi-norm of an $\mathcal{H C} \mathcal{T}$ trace of $\nabla v$, which is a piecewise quadratic vector-function in one independent variable. We will establish in this section an extension estimate for such functions in terms of the $\frac{1}{2}$-order Sobolev semi-norm.

Let $\ldots<a_{-1}<a_{0}=0<a_{1}<a_{2}<\ldots<a_{N}=L<a_{N+1}<\ldots$ be a quasi-uniform subdivision of $\mathbb{R}$, i.e.,

$$
\left|a_{i+1}-a_{i}\right| \approx h
$$

where $h$ is the mesh size of the subdivision. We will denote by $Q_{h}(I)$ the space of continuous piecewise quadratic functions (with respect to the subdivision $a_{0}<a_{1}<\ldots<a_{N}$ ) on 
the interval $I=(0, L)$. Throughout this section, the constant in the statement $F \lesssim G$ is independent of $L$ and $h$.

Let $q \in Q_{h}(I)$. The semi-norm $|q|_{H^{1 / 2}(I)}$ is defined by

$$
|q|_{H^{1 / 2}(I)}^{2}=\int_{0}^{L} \int_{0}^{L} \frac{|q(x)-q(y)|^{2}}{|x-y|^{2}} d x d y
$$

We have the following discrete Sobolev inequality (cf. [18]).

Lemma 5.1. The following estimate holds:

$$
\|q\|_{L^{\infty}(I)} \lesssim\left(1+\ln \frac{L}{h}\right)^{1 / 2}\left[L^{-\frac{1}{2}}\|q\|_{L^{2}(I)}+|q|_{H^{1 / 2}(I)}\right] \quad \forall q \in Q_{h}(I)
$$

The following lemma extends a well-known result for piecewise linear functions (cf. [8]).

Lemma 5.2. Suppose $q \in Q_{h}(I)$ vanishes at the endpoints of $I$, and $\tilde{q}$ is the extension of $q$ to $\mathbb{R}$ that vanishes outside the interval $I$. Then we have

$$
|\tilde{q}|_{H^{1 / 2}(\mathbb{R})}^{2} \lesssim\left(1+\ln \frac{L}{h}\right)\|q\|_{L^{\infty}(I)}^{2}+|q|_{H^{1 / 2}(I)}^{2}
$$

Proof. It follows from (5.2) and a straight forward calculation that

$$
\begin{aligned}
|\tilde{q}|_{H^{1 / 2}(\mathbb{R})}^{2} & =\int_{\mathbb{R}} \int_{\mathbb{R}} \frac{|\tilde{q}(x)-\tilde{q}(y)|^{2}}{|x-y|^{2}} d x d y \\
& =|q|_{H^{1 / 2}(I)}^{2}+2 \int_{0}^{L} \frac{q^{2}(x)}{x} d x+2 \int_{0}^{L} \frac{q^{2}(x)}{L-x} d x .
\end{aligned}
$$

We can write $\int_{0}^{L} \frac{q^{2}(x)}{x} d x=\int_{0}^{a_{1}} \frac{q^{2}(x)}{x} d x+\int_{a_{1}}^{L} \frac{q^{2}(x)}{x} d x$. It is clear that the second integral is bounded by $[1+\ln (L / h)]\|q\|_{L^{\infty}(I)}^{2}$. The first integral is bounded by $\|q\|_{L^{\infty}(I)}^{2}$ by a scaling argument. Similar estimates hold for $\int_{0}^{L} \frac{q^{2}(x)}{L-x} d x$. Therefore

$$
\int_{0}^{L} \frac{q^{2}(x)}{x} d x+\int_{0}^{L} \frac{q^{2}(x)}{L-x} d x \lesssim\left(1+\ln \frac{L}{h}\right)\|q\|_{L^{\infty}(I)}^{2} .
$$

The estimate (5.4) now follows from (5.5) and (5.6).

We now apply the above two basic lemmas to study the extension of continuous piecewise quadratic vector-functions. Note that such functions are determined by their values at the midpoints and endpoints of the subintervals. Given a vector function $\mathbf{q}=$ $\left(q_{1}, q_{2}\right)^{t} \in\left[Q_{h}(I)\right]^{2}$ and $2 \times 2$ matrices $\mathbf{A}_{\ell}$ and $\mathbf{A}_{r}$, we can define the continuous piecewise 
quadratic vector-functions $\mathbf{q}_{\ell}$ and $\mathbf{q}_{r}$ as follows. The function $\mathbf{q}_{\ell}$ vanishes outside the interval $\left(a_{-1}, a_{1}\right)$ and $\left.\mathbf{q}_{\ell}\right|_{\left[a_{-1}, a_{1}\right]}$ is determined by (i) $\mathbf{q}_{\ell}\left(a_{-1}\right)=\mathbf{q}_{\ell}\left(a_{1} / 2\right)=\mathbf{q}_{\ell}\left(a_{1}\right)=\mathbf{0}$, (ii) $\mathbf{q}_{\ell}(0)=\mathbf{q}(0)$, and (iii) $\left[\mathbf{q}_{\ell}\left(a_{-1} / 2\right)\right]=\mathbf{A}_{\ell}[\mathbf{q}(0)]$. Similarly, the function $\mathbf{q}_{r}$ vanishes outside $\left(a_{N-1}, a_{N+1}\right)$, and $\left.\mathbf{q}_{r}\right|_{\left[a_{N-1}, a_{N+1}\right]}$ is determined by (i) $\mathbf{q}_{r}\left(a_{N-1}\right)=\mathbf{q}_{r}\left(\left(a_{N-1}+L\right) / 2\right)=$ $\mathbf{q}_{r}\left(a_{N+1}\right)=\mathbf{0}$, (ii) $\mathbf{q}_{r}(L)=\mathbf{q}(L)$ and (iii) $\left[\mathbf{q}_{\ell}\left(\left(L+a_{N+1}\right) / 2\right)\right]=\mathbf{A}_{r}[\mathbf{q}(L)]$.

We assume that

$$
\left\|\mathbf{A}_{\ell}\right\|_{\infty}+\left\|\mathbf{A}_{r}\right\|_{\infty} \lesssim 1
$$

Lemma 5.3. Let $\mathbf{q}_{\ell}$ and $\mathbf{q}_{r}$ be the functions defined above. Then we have

$$
\left|\mathbf{q}_{\ell}\right|_{H^{1 / 2}(\mathbb{R})}+\left|\mathbf{q}_{r}\right|_{H^{1 / 2}(\mathbb{R})} \lesssim\|\mathbf{q}\|_{L^{\infty}(I)} .
$$

Proof. $\quad$ First observe that we have, by (5.7), $\left\|\mathbf{q}_{\ell}\right\|_{L^{\infty}\left(\left(a_{-1}, a_{1}\right)\right)} \lesssim|\mathbf{q}(0)|$. Applying Lemma 5.2 to $\mathbf{q}_{\ell}$ on $\left(a_{-1}, a_{1}\right)$ we obtain

$$
\left|\mathbf{q}_{\ell}\right|_{H^{1 / 2}(\mathbb{R})} \lesssim|\mathbf{q}(0)|+\left|\mathbf{q}_{\ell}\right|_{H^{1 / 2}\left(\left(a_{-1}, a_{1}\right)\right)} .
$$

A scaling argument shows that $\left|\mathbf{q}_{\ell}\right|_{H^{1 / 2}\left(\left(a_{-1}, a_{1}\right)\right)} \lesssim|\mathbf{q}(0)|$, and hence

$$
\left|\mathbf{q}_{\ell}\right|_{H^{1 / 2}(\mathbb{R})} \lesssim\|\mathbf{q}\|_{L^{\infty}(I)} .
$$

The estimate for $\left|\mathbf{q}_{r}\right|_{H^{1 / 2}(\mathbb{R})}$ is obtained similarly.

The following extension estimate will play a key role in the condition number estimate (cf. Section 6).

Lemma 5.4. Let $\mathbf{q} \in\left[Q_{h}(I)\right]^{2}$ and $\mathbf{A}_{\ell}, \mathbf{A}_{r}$ be $2 \times 2$ matrices satisfying (5.7). Let $\tilde{\mathbf{q}}$ be a continuous piecewise quadratic extension of $\mathbf{q}$ to $\mathbb{R}$ defined by (i) $\tilde{\mathbf{q}}$ vanishes outside $\left(a_{-1}, a_{N+1}\right)$; (ii) $\left.\tilde{\mathbf{q}}\right|_{\left[a_{-1}, 0\right]}$ is determined by $\tilde{\mathbf{q}}\left(a_{-1}\right)=\mathbf{0},\left[\tilde{\mathbf{q}}\left(a_{-1} / 2\right)\right]=\mathbf{A}_{\ell}[\mathbf{q}(0)]$ and $\tilde{\mathbf{q}}(0)=\mathbf{q}(0) ;$ (iii) $\left.\tilde{\mathbf{q}}\right|_{\left[L, a_{N+1}\right]}$ is determined by $\tilde{\mathbf{q}}\left(a_{N+1}\right)=\mathbf{0},\left[\tilde{\mathbf{q}}\left(\left(L+a_{N+1}\right) / 2\right)\right]=\mathbf{A}_{r}[\mathbf{q}(L)]$, and $\tilde{\mathbf{q}}(L)=\mathbf{q}(L)$. Then we have

$$
|\tilde{\mathbf{q}}|_{H^{1 / 2}(\mathbb{R})}^{2} \lesssim\left(1+\ln \frac{L}{h}\right)^{2}\left[L^{-1}\|\mathbf{q}\|_{L^{2}(I)}^{2}+|\mathbf{q}|_{H^{1 / 2}(I)}^{2}\right]
$$

Proof. Let $\mathbf{q}_{\ell}$ and $\mathbf{q}_{r}$ be the functions defined in the paragraph before Lemma 5.3, and the function $\mathbf{q}_{m}$ on $I$ be defined by

$$
\mathbf{q}_{m}=\mathbf{q}-\left.\mathbf{q}_{\ell}\right|_{I}-\left.\mathbf{q}_{r}\right|_{I}
$$

We denote by $\tilde{\mathbf{q}}_{m}$ the extension of $\mathbf{q}_{m}$ to $\mathbb{R}$ that vanishes outside the interval $I$. 
We can express $\tilde{\mathbf{q}}$ as

$$
\tilde{\mathbf{q}}=\mathbf{q}_{\ell}+\tilde{\mathbf{q}}_{m}+\mathbf{q}_{r}
$$

Combining (5.8) and (5.3) we have

$$
\left|\mathbf{q}_{r}\right|_{H^{1 / 2}(\mathbb{R})}^{2}+\left|\mathbf{q}_{\ell}\right|_{H^{1 / 2}(\mathbb{R})}^{2} \lesssim\left(1+\ln \frac{L}{h}\right)\left[L^{-1}\|\mathbf{q}\|_{L^{2}(I)}^{2}+|\mathbf{q}|_{H^{1 / 2}(I)}^{2}\right] .
$$

Since $\mathbf{q}$ vanishes at the endpoints of $I$, it follows from (5.4) that

$$
\left|\tilde{\mathbf{q}}_{m}\right|_{H^{1 / 2}(\mathbb{R})}^{2} \lesssim\left(1+\ln \frac{L}{h}\right)\left\|\mathbf{q}_{m}\right\|_{L^{\infty}(I)}^{2}+\left|\mathbf{q}_{m}\right|_{H^{1 / 2}(I)}^{2} .
$$

Using (5.12) and the fact that $\left\|\mathbf{q}_{\ell}\right\|_{L^{\infty}(\mathbb{R})} \lesssim|\mathbf{q}(0)|$ and $\left\|\mathbf{q}_{r}\right\|_{L^{\infty}(\mathbb{R})} \lesssim|\mathbf{q}(L)|$, we have

$$
\left\|\mathbf{q}_{m}\right\|_{L^{\infty}(I)} \lesssim\|\mathbf{q}\|_{L^{\infty}(I)}
$$

Also, Lemma 5.3 and (5.12) imply that

$$
\begin{aligned}
\left|\mathbf{q}_{m}\right|_{H^{1 / 2}(I)} & \lesssim|\mathbf{q}|_{H^{1 / 2}(I)}+\left|\mathbf{q}_{\ell}\right|_{H^{1 / 2}(I)}+\left|\mathbf{q}_{r}\right|_{H^{1 / 2}(I)} \\
& \lesssim|\mathbf{q}|_{H^{1 / 2}(I)}+\|\left.\mathbf{q}\right|_{L^{\infty}(I)}
\end{aligned}
$$

Combining (5.15)-(5.17) and (5.3) we obtain

$$
\left|\tilde{\mathbf{q}}_{m}\right|_{H^{1 / 2}(\mathbb{R})}^{2} \lesssim\left(1+\ln \frac{L}{h}\right)^{2}\left[L^{-1}\|\mathbf{q}\|_{L^{2}(I)}^{2}+|\mathbf{q}|_{H^{1 / 2}(I)}^{2}\right] .
$$

The estimate (5.11) now follows from (5.13), (5.14) and (5.18).

\section{Condition Number Estimate}

First we show that the second inequality in (2.23) holds for $C_{2}=1$.

Lemma 6.1. The following estimate holds.

$$
\inf _{\substack{v=v_{0}+\sum_{j=1}^{J} \mathrm{I}_{j} v_{j} \\ v_{0} \in \mathcal{M}_{H}, v_{j} \in \mathcal{M}_{h}\left(\Gamma_{j}\right)}}\left[\left\langle\mathbf{S}_{0} v_{0}, v_{0}\right\rangle+\sum_{j=1}^{J}\left\langle\mathscr{S}_{j} v_{j}, v_{j}\right\rangle\right] \leq\langle\mathbf{S} v, v\rangle \quad \forall v \in \mathcal{M}_{h}(\Gamma) .
$$

Proof. Given any $v \in \mathcal{M}_{h}(\Gamma)$, we define $v_{0}=\mathbf{P}_{0} v \in \mathcal{M}_{H}$ and $\hat{v}=\left(\mathbf{I d}-\mathbf{P}_{0}\right) v$. The functions $v_{j} \in \dot{\mathcal{M}}_{h}\left(\Gamma_{j}\right)$ are defined by

$$
a_{h, j}\left(v_{j}, w\right)=a_{h, j}\left(\mathbf{R}_{j} \hat{v}, w\right) \quad \forall w \in \dot{\mathcal{M}}_{h}\left(\Gamma_{j}\right) .
$$


It follows easily from (6.2) that

$$
\begin{aligned}
& \mathbf{R}_{j} \hat{v}-v_{j} \in Z_{j}, \\
& a_{h, j}\left(v_{j}, v_{j}\right) \leq a_{h, j}\left(\mathbf{R}_{j} \hat{v}, \mathbf{R}_{j} \hat{v}\right) .
\end{aligned}
$$

Using (2.12), (2.13), (2.17), (6.3) and the fact that $\mathbf{P}_{0}$ is a projection operator onto $\mathcal{M}_{H}$, we obtain the following splitting of $v$ :

$$
\begin{aligned}
v & =v_{0}+\left(\mathbf{I d}-\mathbf{P}_{0}\right) \hat{v} \\
& =v_{0}+\sum_{j=1}^{J}\left(\mathbf{I} \mathbf{d}-\mathbf{P}_{0}\right) \mathbf{E}_{j} \mathbf{D}_{j} v_{j}+\left(\mathbf{I} \mathbf{d}-\mathbf{P}_{0}\right) \sum_{j=1}^{J} \mathbf{E}_{j} \mathbf{D}_{j}\left(\mathbf{R}_{j} \hat{v}-v_{j}\right) \\
& =\mathbf{I}_{0} v_{0}+\sum_{j=1}^{J} \mathbf{I}_{j} v_{j} .
\end{aligned}
$$

From $(2.7)-(2.9),(2.11),(2.14),(6.4)$ and the definitions of $v_{0}$ and $\hat{v}$, we have

$$
\begin{aligned}
\left\langle\mathbf{S}_{0} v_{0}, v_{0}\right\rangle+\sum_{j=1}^{J}\left\langle\mathscr{S}_{j} v_{j}, v_{j}\right\rangle & =a_{h}\left(v_{0}, v_{0}\right)+\sum_{j=1}^{J} a_{h, j}\left(v_{j}, v_{j}\right) \\
& \leq a_{h}\left(v_{0}, v_{0}\right)+\sum_{j=1}^{J} a_{h, j}\left(\mathbf{R}_{j} \hat{v}, \mathbf{R}_{j} \hat{v}\right) \\
& =a_{h}\left(v_{0}, v_{0}\right)+a_{h}(\hat{v}, \hat{v}) \\
& =a_{h}\left(v_{0}+\hat{v}, v_{0}+\hat{v}\right)=\langle\mathbf{S} v, v\rangle,
\end{aligned}
$$

which implies (6.1).

Next we estimate the constant $C_{1}$ in $(2.23)$.

Lemma 6.2. The following estimate holds.

$$
\left(1+\ln \frac{H}{h}\right)^{-2}\langle\mathbf{S} v, v\rangle \lesssim \inf _{\substack{v=v_{0}+\sum_{j=1}^{J} \mathrm{I}_{j} v_{j} \\ v_{0} \in \mathcal{M}_{H}, v_{j} \in \mathcal{M}_{h}\left(\Gamma_{j}\right)}}\left[\left\langle\mathbf{S}_{0} v_{0}, v_{0}\right\rangle+\sum_{j=1}^{J}\left\langle\mathscr{S}_{j} v_{j}, v_{j}\right\rangle\right]
$$

Proof. Let $v_{0} \in \mathcal{M}_{H}, v_{j} \in \dot{\mathcal{M}}_{h}\left(\Gamma_{j}\right)$ for $1 \leq j \leq J$, and $v=v_{0}+\sum_{j=1}^{J} \mathbf{I}_{j} v_{j}=v_{0}+(\mathbf{I d}-$ $\left.\mathbf{P}_{0}\right) \sum_{j=1}^{J} \mathbf{E}_{j} \mathbf{D}_{j} v_{j}$. Since $\mathbf{P}_{0}$ is the $a_{h}(\cdot, \cdot)$-orthogonal projection operator onto $\mathcal{M}_{H}$, we derive from (2.7), Pythagoras' theorem and the arithmetic-geometric inequality that

$$
\begin{aligned}
\langle\mathbf{S} v, v\rangle & =a_{h}\left(v_{0}, v_{0}\right)+a_{h}\left(\left(\mathbf{I} \mathbf{d}-\mathbf{P}_{0}\right) \sum_{j=1}^{J} \mathbf{E}_{j} \mathbf{D}_{j} v_{j},\left(\mathbf{I d}-\mathbf{P}_{0}\right) \sum_{j=1}^{J} \mathbf{E}_{j} \mathbf{D}_{j} v_{j}\right) \\
& \leq\left\langle\mathbf{S}_{0} v_{0}, v_{0}\right\rangle+a_{h}\left(\sum_{j=1}^{J} \mathbf{E}_{j} \mathbf{D}_{j} v_{j}, \sum_{j=1}^{J} \mathbf{E}_{j} \mathbf{D}_{j} v_{j}\right) \\
& \lesssim\left\langle\mathbf{S}_{0} v_{0}, v_{0}\right\rangle+\sum_{j=1}^{J} a_{h}\left(\mathbf{E}_{j} \mathbf{D}_{j} v_{j}, \mathbf{E}_{j} \mathbf{D}_{j} v_{j}\right)
\end{aligned}
$$


Since $v_{j} \in \dot{\mathcal{M}}_{h}\left(\Gamma_{j}\right)$ vanishes at the cross points of $\Omega_{j}$, the function $\mathbf{E}_{j} \mathbf{D}_{j} v_{j}$ vanishes on all the subdomains except those $\Omega_{\ell}$ such that $\Omega_{j}$ and $\Omega_{\ell}$ share (part of) a side. We will denote the index set for such subdomains by $\mathcal{N}_{j}$. Note that the shape regularity assumptions (4.1)-(4.2) imply that $\left|\mathcal{N}_{j}\right|$ is bounded by a constant independent of $H, h$ and $J$, which justifies the last step in $(6.8)$.

In view of (2.9) we can write

$$
a_{h}\left(\mathbf{E}_{j} \mathbf{D}_{j} v_{j}, \mathbf{E}_{j} \mathbf{D}_{j} v_{j}\right)=\sum_{\ell \in \mathcal{N}_{j}} a_{h, \ell}\left(\mathbf{R}_{\ell} \mathbf{E}_{j} \mathbf{D}_{j} v_{j}, \mathbf{R}_{\ell} \mathbf{E}_{j} \mathbf{D}_{j} v_{j}\right) .
$$

It remains only to estimate $a_{h, \ell}\left(\mathbf{R}_{\ell} \mathbf{E}_{j} \mathbf{D}_{j} v_{j}, \mathbf{R}_{\ell} \mathbf{E}_{j} \mathbf{D}_{j} v_{j}\right)$ for each $\ell \in \mathcal{N}_{j}$.

Recall that functions in $\dot{\mathcal{M}}_{h}\left(\Gamma_{j}\right)$ vanishes at the cross points of $\Omega_{j}$ and hence

$$
\mathbf{D}_{j} v_{j}=\frac{v_{j}}{2} \quad \forall v_{j} \in \dot{\mathcal{M}}_{h}\left(\Gamma_{j}\right) \text {. }
$$

It therefore suffices to estimate $a_{h, \ell}\left(\mathbf{R}_{\ell} \mathbf{E}_{j} v_{j}, \mathbf{R}_{\ell} \mathbf{E}_{j} v_{j}\right)$ by $a_{h, j}\left(v_{j}, v_{j}\right)$, and we may assume $\ell \neq j$ since the case $\ell=j$ is trivial.

Let $e$ be the common side of $\Omega_{j}$ and $\Omega_{\ell}$, and $v_{j, \ell}=\mathbf{R}_{\ell} \mathbf{E}_{j} v_{j}$. Observe that, by the definition of $\mathbf{E}_{j}$ and (3.1), $\nabla v_{j, \ell}$ vanishes at all the midpoints of $\partial \Omega_{j}$ that are not on $e$, since $v_{j}$ vanishes at the endpoints of $e$. Using the freedom in choosing the adjacent midpoint in $(3.2 c)$, we can construct $\mathcal{H C} \mathcal{T}$ conforming traces $\left(\mathscr{D}_{1} v_{j}, \mathscr{D}_{2} v_{j}\right)$ and $\left(\mathscr{D}_{1} v_{j, \ell}, \mathscr{D}_{2} v_{j, \ell}\right)$ for $\nabla v_{j}$ and $\nabla v_{j, \ell}$ such that

$$
\left(\left.\mathscr{D}_{1} v_{j}\right|_{e},\left.\mathscr{D}_{2} v_{j}\right|_{e}\right)=\left(\left.\mathscr{D}_{1} v_{j, \ell}\right|_{e},\left.\mathscr{D}_{2} v_{j, \ell}\right|_{e}\right),
$$

$\left(\mathscr{D}_{1} v_{j, \ell}, \mathscr{D}_{2} v_{j, \ell}\right) \quad$ vanishes at all vertices and midpoints that are not on $e$, except the two midpoints next to the endpoints of $e$ (cf. Figure 4).

Observe that the identity

$$
g^{\prime}((a+b) / 2)=\frac{3}{2}\left(\frac{g(b)-g(a)}{b-a}\right)-\left(\frac{g^{\prime}(a)+g^{\prime}(b)}{4}\right)
$$

holds for any cubic polynomial. Since $v_{j} \in \dot{\mathcal{M}}_{h}\left(\Gamma_{j}\right)$ vanishes at the vertices of $e$, we have the following consequence of (6.13).

(6.14) Let $m$ be a midpoint on $\partial \Omega_{\ell} \backslash e$ which is next to the vertex $p$ of $e$ (cf. Figure 4). Then $\left(\mathscr{D}_{1} v_{j, \ell}\right)(m)$ and $\left(\mathscr{D}_{2} v_{j, \ell}\right)(m)$ are linear combinations of $\left(\mathscr{D}_{1} v_{j}\right)(p)$ and $\left(\mathscr{D}_{2} v_{j}\right)(p)$, with coefficients bounded by universal constants.

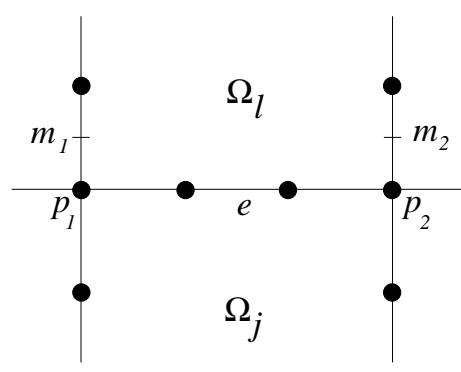

Figure 4 
Recall that $\mathcal{H C} \mathcal{T}$ conforming traces are piecewise quadratic vector-functions. It follows from (6.11)-(6.12), (6.14) and Lemma 5.4 that

$$
\begin{aligned}
\left|\mathscr{D}_{1} v_{j, \ell}\right|_{H^{1 / 2}\left(\partial \Omega_{\ell}\right)}^{2} & \lesssim\left(1+\ln \frac{H}{h}\right)^{2}\left[\left|\mathscr{D}_{1} v\right|_{H^{1 / 2}\left(\partial \Omega_{j}\right)}^{2}+H^{-1}\left\|\mathscr{D}_{1} v\right\|_{L^{2}\left(\partial \Omega_{j}\right)}^{2}\right] \\
\left|\mathscr{D}_{2} v_{j, \ell}\right|_{H^{1 / 2}\left(\partial \Omega_{\ell}\right)}^{2} & \lesssim\left(1+\ln \frac{H}{h}\right)^{2}\left[\left|\mathscr{D}_{2} v\right|_{H^{1 / 2}\left(\partial \Omega_{j}\right)}^{2}+H^{-1}\left\|\mathscr{D}_{2} v\right\|_{L^{2}\left(\partial \Omega_{j}\right)}^{2}\right] .
\end{aligned}
$$

Since $\left(\mathscr{D}_{1} v_{j}, \mathscr{D}_{2} v_{j}\right)$ (resp. $\left.\left(\mathscr{D}_{1} v_{j, \ell}, \mathscr{D}_{2} v_{j, \ell}\right)\right)$ is the restriction of the gradient of a function in $\mathcal{H C} \mathcal{T}_{h}\left(\bar{\Omega}_{j}\right)$ (resp. $\mathcal{H C} \mathcal{T}_{\ell}\left(\bar{\Omega}_{\ell}\right)$ ) which vanishes at all the vertices of $\Omega_{j}$ (resp. $\Omega_{\ell}$ ), it follows from Lemma 4.8 and $(6.15)-(6.16)$ that

$$
\begin{aligned}
& \left|\mathscr{D}_{1} v_{j, \ell}\right|_{H^{1 / 2}\left(\partial \Omega_{\ell}\right)}^{2}+\left|\mathscr{D}_{2} v_{j, \ell}\right|_{H^{1 / 2}\left(\partial \Omega_{\ell}\right)}^{2} \\
\lesssim & \left(1+\ln \frac{H}{h}\right)^{2}\left[\left|\mathscr{D}_{1} v\right|_{H^{1 / 2}\left(\partial \Omega_{j}\right)}^{2}+\left|\mathscr{D}_{2} v\right|_{H^{1 / 2}\left(\partial \Omega_{j}\right)}^{2}\right] .
\end{aligned}
$$

From Lemma 4.6, (4.16) and (6.17) we obtain

$$
a_{h, \ell}\left(\mathbf{R}_{\ell} \mathbf{E}_{j} v_{j}, \mathbf{R}_{\ell} \mathbf{E}_{j} v_{j}\right) \lesssim\left(1+\ln \frac{H}{h}\right)^{2} a_{h, j}\left(v_{j}, v_{j}\right)
$$

The estimate (6.7) now follows from (6.8)-(6.10), (6.18) and (2.11).

Combining Lemmas 2.5, 6.1 and 6.2, we have the following theorem on the condition number of the operator BS.

Theorem 6.3. There exists a constant $C>0$ independent of $h, H$, and $J$, such that

$$
\frac{\lambda_{\max }(\mathbf{B S})}{\lambda_{\min }(\mathbf{B S})} \leq C\left(1+\ln \frac{H}{h}\right)^{2}
$$

Remark 6.4. Let the square $\Omega$ with vertices $(-1,-1),(2,-1),(2,2)$ and $(-1,2)$ be partitioned into nine unit squares, and $\mathcal{T}_{h}$ be a uniform triangulation of $\Omega$ with mesh size $h$. Let $\Omega_{1}$ be the subdomain with vertices $(0,0),(1,0),(1,1)$ and $(0,1)$, and $v$ be the discrete biharmonic Morley function on $\Omega_{1}$ whose nodal values on $\partial \Omega_{1}$ coincide with those defined by the function $\tilde{v}=x_{1} x_{2}$. Clearly $\tilde{v}$ is an $\mathcal{H C} \mathcal{T}$ relative of $v$ and $\nabla \tilde{v}=\left(x_{2}, x_{1}\right)$ is an $\mathcal{H C} \mathcal{T}$ trace of $\nabla v$. Let $\Omega_{2}$ be the neighboring subdomain with vertices $(0,1),(1,1)$, $(1,2)$ and $(0,2)$. Since $v(1,1)=1$, Lemma 4.6 and the identity $(6.13)$ imply that the nonconforming $|\cdot|_{H^{2}\left(\mathcal{T}_{h}\right)}$ semi-norm of the extension of $v$ to $\Omega_{2}$ is of order $h^{-1}$. On the other hand the nonconforming $|\cdot|_{H^{2}\left(\mathcal{T}_{h}\right)}$ semi-norm of $v$ on $\Omega_{1}$ is of order 1 by Lemma 4.6. It follows that for this $\Omega$ the condition number of KS (cf. Remark 2.4) is of order $h^{-2}$ (cf. Lemma 2.5). Hence, the condition number of $\mathbf{K S}$ can in general grow at the rate of $(H / h)^{2}$.

Remark 6.5. The BDD method for nonconforming plate elements can also be applied to plates where the physical constants have jumps across subdomains. The estimate (6.19) 
is still valid, where $C$ is also independent of the jumps of the physical constants. This is achieved by choosing appropriate diagonal scaling operators $\mathbf{D}_{j}$ which take into account such jumps, as in the conforming case (cf. [28], [25], and [26]).

\section{Numerical Results}

We have carried out three numerical experiments. The first two experiments are performed for a rectangular plate whose vertices are located at $(0,0),(4,0),(4,3)$ and $(0,3)$. It is divided into 12 subdomains (cf. Figure 5) for the first experiment and 48 subdomains (cf. Figure 6) for the second experiment. The third experiment is performed for an L-shaped plate whose vertices are located at $(0,0),(0,2),(-2,2),(-2,-2),(2,-2)$ and $(2,0)$, and it is divided into 8 subdomains (cf. Figure 7 ). In all three experiments the plates are triangulated uniformly by triangles whose sides have slopes $0,-1$ and $\infty$, and the mesh size $h$ is taken to be the length of the horizontal edges of the triangles. The Poisson ratio $\nu$ equals 0.3 in all experiments.

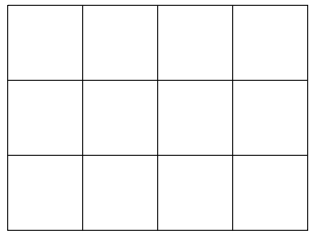

Figure 5

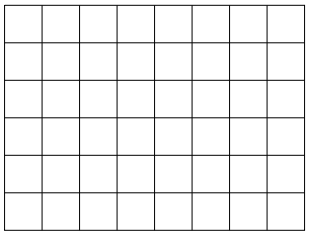

Figure 6

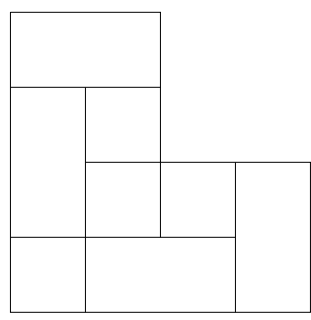

Figure 7

The condition numbers $\kappa(\mathbf{S}), \kappa(\mathbf{K S})$ and $\kappa(\mathbf{B S})$ are displayed in Table 1, Table 2 and Table 3, where $\mathbf{S}$ is the Schur complement with respect to the skeleton of the subdomains, $\mathbf{K}$ is the preconditioner where the coarse grid space is constructed by using the kernels of the subdomain Schur complements (cf. Remark 2.4), and B is the BDD preconditioner defined in (2.18). In all three experiments $\kappa(\mathbf{S}), \kappa(\mathbf{K S})$ and $\kappa(\mathbf{B S})$ grow at the rates of $h^{-3}, h^{-2}$ and $|\ln h|^{2}$ respectively. The results of the first two experiments also indicate that the constant $C$ in (6.19) is indeed independent of the number of subdomains.

\begin{tabular}{|c|c|c|c|}
\hline$h$ & $\kappa(\mathbf{S})$ & $\kappa(\mathbf{K S})$ & $\kappa(\mathbf{B S})$ \\
\hline \hline$(0.5)^{0}$ & $8.611 \times 10^{0}$ & $2.262 \times 10^{0}$ & $1.062 \times 10^{0}$ \\
\hline$(0.5)^{1}$ & $3.238 \times 10^{1}$ & $6.288 \times 10^{0}$ & $1.657 \times 10^{0}$ \\
\hline$(0.5)^{2}$ & $2.046 \times 10^{2}$ & $2.325 \times 10^{1}$ & $3.104 \times 10^{0}$ \\
\hline$(0.5)^{3}$ & $1.522 \times 10^{3}$ & $9.211 \times 10^{1}$ & $5.446 \times 10^{0}$ \\
\hline$(0.5)^{4}$ & $1.209 \times 10^{4}$ & $3.682 \times 10^{2}$ & $8.323 \times 10^{0}$ \\
\hline
\end{tabular}

Table 1. Rectangular Plate (12 Subdomains) 


\begin{tabular}{|c|c|c|c|}
\hline$h$ & $\kappa(\mathbf{S})$ & $\kappa(\mathbf{K S})$ & $\kappa(\mathbf{B S})$ \\
\hline \hline$(0.5)^{1}$ & $6.501 \times 10^{1}$ & $1.595 \times 10^{0}$ & $1.038 \times 10^{0}$ \\
\hline$(0.5)^{2}$ & $3.595 \times 10^{2}$ & $6.066 \times 10^{0}$ & $1.761 \times 10^{0}$ \\
\hline$(0.5)^{3}$ & $2.849 \times 10^{3}$ & $2.139 \times 10^{1}$ & $3.543 \times 10^{0}$ \\
\hline$(0.5)^{4}$ & $2.308 \times 10^{4}$ & $8.228 \times 10^{1}$ & $6.316 \times 10^{0}$ \\
\hline
\end{tabular}

Table 2. Rectangular Plate (48 Subdomains)

\begin{tabular}{|c|c|c|c|}
\hline$h$ & $\kappa(\mathbf{S})$ & $\kappa(\mathbf{K S})$ & $\kappa(\mathbf{B S})$ \\
\hline \hline$(0.5)^{0}$ & $6.732 \times 10^{0}$ & $2.234 \times 10^{0}$ & $1.015 \times 10^{0}$ \\
\hline$(0.5)^{1}$ & $1.538 \times 10^{1}$ & $5.025 \times 10^{0}$ & $1.585 \times 10^{0}$ \\
\hline$(0.5)^{2}$ & $8.407 \times 10^{1}$ & $1.828 \times 10^{1}$ & $2.784 \times 10^{0}$ \\
\hline$(0.5)^{3}$ & $5.875 \times 10^{2}$ & $7.186 \times 10^{1}$ & $4.645 \times 10^{0}$ \\
\hline$(0.5)^{4}$ & $4.547 \times 10^{3}$ & $2.860 \times 10^{2}$ & $7.072 \times 10^{0}$ \\
\hline
\end{tabular}

Table 3. L-shaped Plate

Acknowledgment Part of the research for this paper was carried out while the first author was visiting the Insititute for Mathematics and Its Applications at the University of Minnesota. She would like to thank the IMA for their support and hospitality.

\section{References}

1. A.Adini and R.W. Clough, Analysis of plate bending by the finite element method, NSF Report G. 7337 (1961).

2. J.H. Argyris, I. Fried and D.W. Scharpf, The TUBA family of plate elements for the matrix displacement method, Aero. J. Roy. Aero. Soc. 72 (1968), 701-709.

3. G.P. Bazeley, Y.K. Cheung, B.M. Irons, and O.C. Zienkiewicz, Triangular elements in bending-conforming and nonconforming solutions, Proceedings of the Conference on Matrix Methods in Structural Mechanics (1965), Wright Patterson A.F.B., Ohio.

4. K. Bell, A refined triangular plate bending element, Internat. J. Numer. Methods Engrg. 1 (1969), 101-122.

5. P.E. Bjørstad and O.B. Widlund, Iterative methods for the solution of elliptic problems on regions partitioned into substructures, SIAM J. Numer. Anal. 23 (1986), 1093-1120.

6. F.K. Bogner, R.L. Fox and L.A. Schmit, The generation of interelement compatible stiffness and mass matrices by the use of interpolation formulas, Proceedings of the Conference on Matrix Methods in Structural Mechanics (1965), Wright Patterson A.F.B., Ohio.

7. J.H. Bramble and S.R. Hilbert, Estimation of linear functionals on Sobolev spaces with application to Fourier transforms and spline interpolation, SIAM J. Numer. Anal. 7 (1970), 113-124. 
8. J.H. Bramble, J.E. Pasciak and A.H. Schatz, The construction of preconditioners for elliptic problems by substructuring I, Math. Comp. 47 (1986), 103-134.

9. S.C. Brenner, Two-level additive Schwarz preconditioners for nonconforming finite elements, Domain Decomposition Methods in Scientific and Engineering Computing, Contemporary Mathematics 180 (D.E. Keyes et. al., eds), American Mathematical Society, Providence, 1994, 9-14.

10. S.C. Brenner, Two-level additive Schwarz preconditioners for nonconforming finite element methods, Math. Comp. 65 (1996), 897-921.

11. S.C. Brenner, A two-level additive Schwarz preconditioner for nonconforming plate elements, Numer. Math. 72 (1996), 419-447.

12. S.C. Brenner, Two-level additive Schwarz preconditioners for plate elements, Wuhan University Journal of Natural Sciences, 1 (1996), 658-667.

13. S.C. Brenner and L.R. Scott, The Mathematical Theory of Finite Element Methods, Springer-Verlag, New York-Berlin-Heidelberg, 1994.

14. P.G. Ciarlet, The Finite Element Method for Elliptic Problems, North-Holland, Amsterdam-New York-Oxford, 1978.

15. R.W. Clough and J.L. Tocher, Finite element stiffness matrices for analysis of plates in bending, Proceedings of the Conference on Matrix Methods in Structural Mechanics, Wright Patterson A.F.B., Ohio, 1965.

16. L.C. Cowsar, Domain decomposition methods for nonconforming finite elements spaces of Lagrange type, Proceedings of the Sixth Copper Mountain Conference on Multigrid Methods, NASA Conference Publication 3224 (1993), 93-109.

17. L.C. Cowsar, J. Mandel and M.F. Wheeler, Balancing domain decomposition for mixed finite elements, Math. Comp. 64 (1995), 989-1015.

18. M. Dryja, A method of domain decomposition for three dimensional finite element elliptic problems, in First International Symposium on Domain Decomposition Methods for Partial Differential Equations (Paris 1987), R. Glowinski, G. H. Golub, G. Meurant and J. Périaux, eds., SIAM, Philadelphia 1988, 43-61.

19. M. Dryja and O.B. Widlund, Some domain decomposition algorithms for elliptic problems, Technical Report 438, Department of Computer Science Courant Institute, 1989.

20. M. Dryja and O.B. Widlund, Schwarz methods of Neumann-Neumann type for threedimensional elliptic finite element problems, Comm. Pure Appl. Math. 48 (1995), $121-155$

21. B. Fraeijs de Veubeke, A conforming finite element for plate bending, Int. J. Solids and Structure 4 (1968), 95-108.

22. B. Fraeijs de Veubeke, Variational principles and the patch test, Internat. J. Numer. Methods Engrg. 8 (1974), 738-801.

23. M. Griebel and P. Oswald, On the abstract theory of additive and multiplicative Schwarz algorithms, Numer. Math. 70 (1995), 163-180.

24. P. Grisvard, Elliptic Problems in Nonsmooth Domains, Pitman, Boston, 1985. 
25. P. Le Tallec, J. Mandel and M. Vidrascu, Balancing domain decomposition for plates, Domain Decomposition Methods in Scientific and Engineering Computing, Contemporary Mathematics 180 (D.E. Keyes et. al., eds), American Mathematical Society, Providence, 1994, 515-524.

26. P. Le Tallec, J. Mandel and M. Vidrascu, A Neumann-Neumann domain decomposition algorithm for solving plate and shell problems, preprint (1997).

27. J. Mandel, Balancing domain decomposition, Comm. Numer. Methods Engrg. 9 (1993), 233-241.

28. J. Mandel and M. Brezina, Balancing domain decomposition for problems with large jumps in coefficients, Math. Comp. 65 (1996), 1387-1401.

29. L.S.D. Morley, The triangular equilibrium problem in the solution of plate bending problems, Aero. Quart. 19 (1968), 149-169.

30. J. Nečas, Les Méthodes Directes en Théorie des Equations Elliptiques, Masson, Paris, 1967.

31. S.V. Nepomnyaschikh, On the application of the bordering method to the mixed boundary value problem for elliptic equations and on mesh norms in $W^{1 / 2}(S)$, Sov. J. Numer. Anal. Math. Modelling, 4 (1989), 493-506.

32. S.V. Nepomnyaschikh, Fictitious components and subdomain alternating methods, Sov. J. Numer. Anal. Math. Modelling 5 (1990), 53-68.

33. L.R. Scott and S. Zhang, Finite element interpolation of non-smooth functions satisfying boundary conditions, Math. Comp. 54 (1990), 483-493.

34. Z. Shi, On the convergence of the incomplete biquadratic nonconforming plate element, Math. Numer. Sinica 8 (1986), 53-62 (in Chinese).

35. Z. Shi and Z. Xie, Substructure preconditioners for nonconforming plate elements, in Domain Decomposition Methods in Science and Engineering, R. Glowinski, J. Peeriaux and Z. Shi, eds., John Wiley and Sons, 1997, 109-115.

36. B. Smith, P. Bjørstad and W. Gropp, Domain Decomposition, Cambridge University Press, Cambridge, 1996.

37. O. Widlund, An extension theorem for finite element spaces with three applications, in Numerical Techniques in Continuum Mechanics (Proceedings of the Second GAMMSeminar, Kiel, 1986), W. Hackbusch and K. Witsch, eds., Braunschweig/Wiesbaden, Notes on Numerical Fluid Mechanics, v. 16, Vieweg, 1987, 110-122.

38. X. Zhang, Studies in Domain Decomposition: Multi-level Methods and the Biharmonic Dirichlet Problem, Dissertation, (Technical Report 584, Department of Computer Science) Courant Institute, 1991.

Susanne C. Brenner

Department of Mathematics

University of South Carolina

Columbia, SC 29208

brenner@math.sc.edu
Li-yeng Sung

Department of Mathematics

University of South Carolina

Columbia, SC 29208

sung@math.sc.edu 